\title{
ISOTOPIC TITANIUM ABUNDANCES IN LOCAL M DWARFS
}

\author{
Joy Chavez ${ }^{1}$ AND David L. LAMBerT ${ }^{2}$ \\ ${ }^{1}$ Department of Astronomy, University of Texas, 1 University Station, C1400 Austin, TX 78712-0259, USA; jchavez@astro.as.utexas.edu \\ 2 McDonald Observatory, University of Texas at Austin, 1 University Station, C1402 Austin, TX 78712-0259, USA; dll@astro.as.utexas.edu \\ Received 2008 September 12; accepted 2009 May 15; published 2009 June 26
}

\begin{abstract}
Relative abundances of the five stable isotopes of titanium $\left({ }^{46} \mathrm{Ti}\right.$ to $\left.{ }^{50} \mathrm{Ti}\right)$ are measured for $11 \mathrm{M}$ dwarfs belonging to the thin disk (four stars), thick disk (three stars), the halo (one star), and either the thick or the thin disk (three stars). Over the metallicity range of the sample $(-1<[\mathrm{Fe} / \mathrm{H}]<0)$, the isotopic ratios are approximately constant at the solar system ratios. There is no discernible difference between the isotopic ratios for thin and thick disk stars. Isotopic ratios are in fair accord with recent calculations of Galactic chemical evolution despite the fact that such calculations underpredict $[\mathrm{Ti} / \mathrm{Fe}]$ by about $0.4 \mathrm{dex}$ at all metallicities.
\end{abstract}

Key word: stars: abundances

Online-only material: color figures

\section{INTRODUCTION}

Insights into the chemical evolution of the Galaxy are provided by the relative abundances of elements in stars belonging to the main populations of the Galaxy: halo, disk (thick and thin), and bulge. In a common representation of abundances, one plots $[\mathrm{X} / \mathrm{Fe}]$ versus $[\mathrm{Fe} / \mathrm{H}]$ for the element $\mathrm{X}$ and $\mathrm{Fe} .{ }^{3}$ Elements may be grouped into classes with each element in a class exhibiting a similar behavior. Comprising one such class are the $\alpha$ elements: the elements in this class include $\mathrm{Mg}, \mathrm{Si}, \mathrm{S}$, and $\mathrm{Ca}$, each with its most abundant isotope having a mass number that is a multiple of 4 . Observations show that the runs of $[\mathrm{X} / \mathrm{Fe}]$ for each of the $\alpha$ elements are similar for stars of a given population presently in the solar neighborhood. The value of $[\mathrm{X} / \mathrm{Fe}]$ is positive and constant (say, $+0.3 \mathrm{dex}$ ) for $[\mathrm{Fe} / \mathrm{H}]<-1$ but decreases to the solar value (i.e., $[\mathrm{X} / \mathrm{Fe}]=0$ ) as thin disk stars are sampled at $[\mathrm{Fe} / \mathrm{H}]>-1$. The change in the variation of $[\mathrm{X} / \mathrm{Fe}]$ versus $[\mathrm{Fe} / \mathrm{H}]$ for $\alpha$ elements at $[\mathrm{Fe} / \mathrm{H}] \simeq-1$ is widely attributed to the onset of contamination of interstellar gas with the Fe-rich $\alpha$-poor ejecta from Type Ia supernovae that reduces the $[\alpha / \mathrm{Fe}]$ previously established by $\alpha$-rich $\mathrm{Fe}$-poor ejecta from Type II supernovae. Theoretical modeling of Galactic chemical evolution (GCE) reproduces quite well the $[\alpha / \mathrm{Fe}]$ versus $[\mathrm{Fe} / \mathrm{H}]$ trends for the standard $\alpha$ elements - see, for example, Timmes et al. (1995, hereafter TWW95), Goswami \& Prantzos (2000, hereafter GP00), and Kobayashi et al. (2006, hereafter K06). Such modeling enterprises, however, ignore the observational differences between the runs of $[\mathrm{X} / \mathrm{Fe}]$ versus $[\mathrm{Fe} / \mathrm{H}]$ for thin and thick disk stars.

Titanium with its principal isotope being ${ }^{48} \mathrm{Ti}$ would appear to qualify as an $\alpha$ element. Indeed, the observed run of [Ti/Fe] with $[\mathrm{Fe} / \mathrm{H}]$ is similar to that of a standard $\alpha$ element. Yet, GCE models fail to predict the observed trend. For example, K06 who reproduce satisfactorily the observed trends for $\mathrm{Mg}, \mathrm{Si}$, and $\mathrm{Ca}$ fail to match the trend for Ti; the predicted Ti trend has the form expected for an $\alpha$ element but at all $[\mathrm{Fe} / \mathrm{H}]$, including the solar value, the predicted $[\mathrm{Ti} / \mathrm{Fe}]$ is about 0.4 dex below the observed trend. GP00 and TWW95 report comparable discrepancies: the predicted $[\mathrm{Ti} / \mathrm{Fe}]$ are $0.3-0.5$ dex below the observations for models that predict satisfactorily the run of $[\mathrm{Ca} / \mathrm{Fe}]$ with $[\mathrm{Fe} / \mathrm{H}]$.

\footnotetext{
3 Standard notation is used here: $[A / B]=\log (A / B)_{\text {star }}-\log (A / B)_{\odot}$ and $\log A \equiv \log \epsilon(A)=\log \left(N_{\mathrm{A}} / N_{\mathrm{H}}\right)+12.00$, where $N$ is the number density.
}

In general, the $\mathrm{K} 06$ predictions are a better fit to observations for elements from $\mathrm{C}$ to $\mathrm{Zn}$ than either the TWW95 or GP00 predictions. These failures to account for the evolution of the Ti abundance are not attributable to inappropriate choices for the reference (i.e., solar) abundances of $\mathrm{Ti}$ and $\mathrm{Fe}$ in confronting predictions with observations. Uncertainties over the solar $\mathrm{Ti}$ and $\mathrm{Fe}$ abundances cannot erase a 0.4 dex difference in $[\mathrm{Ti} / \mathrm{Fe}]$ by revisions of the zero points for $[\mathrm{Ti} / \mathrm{Fe}]$ and $[\mathrm{Fe} / \mathrm{H}]$. For example, substitution of the Asplund et al. (2005) Ti and Fe abundances for those of Anders \& Grevesse (1989) used by K06 shifts K06's predictions by 0.22 dex to higher $[\mathrm{Fe} / \mathrm{H}]$ and by 0.13 dex to higher $[\mathrm{Ti} / \mathrm{Fe}]$, a far cry short of the 0.4 dex failure. Clearly, aspects of Ti nucleosynthesis are not yet understood.

Recent work on the compositions of thick and thin disk stars (e.g., Bensby et al. 2005; Reddy et al. 2006) resolve the previously considered single $[\alpha / \mathrm{Fe}]$ versus $[\mathrm{Fe} / \mathrm{H}]$ relation for disk stars into different relations for thick and thin disk stars. Titanium behaves like $\mathrm{Mg}, \mathrm{Si}$, and $\mathrm{Ca}$ in its $[\mathrm{X} / \mathrm{Fe}]$ versus $[\mathrm{Fe} / \mathrm{H}]$ differences for the thick and thin disks. Modeling of the thick and thin disks has yet to reach the detailed treatments given in the above cited and other papers for the chemical evolution of the halo-disk combination but apportionment of disk stars between the thin and thick disks is not going to solve the 0.4 dex Ti problem.

With the intent of providing novel observational evidence on titanium nucleosynthesis, we have measured relative abundances for the five stable Ti isotopes for a selection of M dwarfs drawn from the halo, thick, and thin disks and spanning iron abundances from the solar value to about $[\mathrm{Fe} / \mathrm{H}]=-1$. The dominant isotope is ${ }^{48} \mathrm{Ti}$, the prospective $\alpha$-nuclide. The other isotopes are ${ }^{46} \mathrm{Ti},{ }^{47} \mathrm{Ti},{ }^{49} \mathrm{Ti}$, and ${ }^{50} \mathrm{Ti}$ with solar system abundances of $8.25 \%, 7.44 \%, 5.41 \%$, and $5.19 \%$, respectively, and with ${ }^{48} \mathrm{Ti}$ accounting for the lion's share at $73.72 \%$ (Lodders 2003). Predictions about the variation of the isotopic abundances with $[\mathrm{Fe} / \mathrm{H}]$ depend primarily on the yields from and the relative frequencies of Type II and Type Ia supernovae.

Pioneering predictions of the variation of the Ti isotopic ratios with $[\mathrm{Fe} / \mathrm{H}]$ were provided by TWW95: relative to ${ }^{48} \mathrm{Ti}$ and the solar system isotopic ratios, the predictions for ${ }^{46} \mathrm{Ti},{ }^{47} \mathrm{Ti}$, ${ }^{49} \mathrm{Ti}$, and ${ }^{50} \mathrm{Ti}$ were factors of 2 too large, 3 too small, spot on, and 2 too small, respectively. Isotopic ratios were predicted to decline with decreasing $[\mathrm{Fe} / \mathrm{H}]$ by factors of 8 for ${ }^{46} \mathrm{Ti}, 6$ for ${ }^{47} \mathrm{Ti}, 2$ for ${ }^{49} \mathrm{Ti}$, and 30 for ${ }^{50} \mathrm{Ti}$ between $[\mathrm{Fe} / \mathrm{H}]=0$ and -1 . 
These predictions and, in particular, more recent examples invite observational tests. Measurements of the isotopic abundances in stars of different metallicities may not resolve directly the 0.4 dex Ti problem but have the potential to suggest directions in which to look for its solution.

In this paper, we derive the isotopic abundances from highresolution spectra of $\mathrm{M}$ dwarfs covering the TiO molecule's $\gamma$-system's $0-0$ band with its leading red-degraded band head at $7054 \AA$. The chosen spectral window was previously used in investigations of sunspot spectra by Lambert \& Mallia (1972), Mira by Wyckoff \& Wehinger (1972), $\alpha$ Tau by Lambert \& Luck (1977), and a sample of late-type dwarfs and giants by Clegg et al. (1979). These previous exploratory analyses provided neither the precision for the isotopic ratios nor the coverage in $[\mathrm{Fe} / \mathrm{H}]$ necessary to subject predictions to a quantitative test. Our results provide the first quantitative tests of the predictions for disk stars.

\section{SELECTION OF STARS}

Three criteria were applied to the selection of the program stars. First, in order that TiO lines in the $\gamma$-system's $0-0$ band be of sufficient strength to provide detectable lines of the four less abundant Ti isotopes, dwarf stars with a spectral type of early-M were chosen. Dwarfs were preferred to giants because their sharp lines allow clear resolution of lines of the different isotopes; giants provide broader lines resulting in a merging of the lines of different isotopes (Clegg et al. 1979). Second, a magnitude limit was necessarily applied in order that an adequate signalto-noise ratio $(\mathrm{S} / \mathrm{N})$ be obtained in a reasonable total exposure time at the resolving power of 120,000 . Third, a probability calculation was applied to identify members of the thin and thick disks and the halo.

The population assignments were made using the probability recipe suggested by Bensby et al. $(2003,2005)$ and applied also by Reddy et al. (2006, see Equations (1) and (2)). The recipe requires (1) the Galactic velocities $U, V, W(U$ is the velocity toward the center of the Galaxy, $V$ the velocity in the direction of Galactic rotation, and $W$ the velocity toward the north Galactic pole) corrected to the local standard of rest, and (2) the mean $U, V, W$ and their dispersions of the thin disk, thick disk, and halo in the solar neighborhood, and (3) the relative stellar densities of the three populations. Reid et al. (2002) provide a catalog of $U, V, W$ heliocentric velocities for $\mathrm{M}$ dwarfs which we correct to the Local Standard of Rest (Dehnen \& Binney 1998). The descriptions of the kinematics and relative densities of the three populations are those adopted by Ramírez et al. (2007).

Table 1 provides the population assignments for our stars. The stars include one halo star, three thick disk stars, four thin disk stars, and three that might belong to either the thin or the thick disk. To be identified with a particular population, we required the membership probability to be greater than $75 \%$. Otherwise, we considered the star to belong to either the thin or the thick disk.

\section{OBSERVATIONS AND DATA REDUCTION}

High-resolution spectra of the $\mathrm{TiO} \gamma$-system's $0-0$ band from $7045 \AA$ to $7094 \AA$ were obtained for the chosen M dwarfs with the W.J. McDonald Observatory's $2.7 \mathrm{~m}$ Harlan J. Smith Telescope and its 2dcoudé spectrograph (Tull et al. 1995) at a resolving power of about 120,000 for all stars except LHS178 for which a resolving power of 60,000 was used. Observations were made in four observing runs between 2006 July and December.
Table 1

Observed Stars

\begin{tabular}{lccc}
\hline \hline \multicolumn{1}{c}{ Star } & $V$ & Sp. Type & Population \\
\hline GJ184 & 9.2 & M0.5V & Thick disk \\
GJ215 & 8.3 & K7V & Thin disk \\
GJ378 & 9.3 & M1V & Thick disk \\
GJ699 & 9.5 & M4V & Thin (65\%)-Thick (33\%) disk \\
GJ701 & 9.9 & M1V & Thin disk \\
GJ725A & 8.9 & M3V & Thin disk \\
GJ880 & 9.6 & M1.5V & Thin disk \\
GJ908 & 10.2 & M1V & Thin (24\%)-Thick (72\%) disk \\
LHS178 & 10.7 & M1V & Halo \\
LHS1226 & 9.5 & M0.5V & Thin (32\%)-Thick (66\%) disk \\
LHS2018 & 7.9 & K7V & Thick disk \\
\hline
\end{tabular}

For the chosen configuration of the cross-dispersed echelle spectrograph, about $25 \AA$ of an order were recorded for about 20 orders with central wavelengths from $6060 \AA$ to $9400 \AA$. Two settings were required to cover the $7045 \AA$ to $7094 \AA$ interval: a blue setting covered $7045 \AA$ to $7073 \AA$, and a red setting covered $7067 \AA$ to $7094 \AA$. Exposures of 30 minutes each were co-added as necessary to realize the desired $\mathrm{S} / \mathrm{N}: \mathrm{S} / \mathrm{N}$ just to the blue of the $7054 \AA$ band head range from 90 for LHS178 to 250 for GJ880. A Th-Ar hollow cathode lamp was observed to provide the wavelength calibration and a measure of the instrumental profile. A rapidly rotating hot star was observed for telluric line removal and correction for the echelle blaze. Standard $\mathrm{IRAF}^{4}$ reduction techniques were used. Since the TiO lines of interest are distributed across the blue and red settings, it was necessary to merge the two spectra to provide a continuous run from shortward of the leading red-degraded band head of the 0-0 band at $7054 \AA$ to about $7090 \AA$. The region 7045-7054 was used to set the continuum. An order in the merged spectrum covering 8420-8470 $\AA$ provides a set of Ti I lines used in the determination of the $\mathrm{Ti}$ abundance.

\section{METHOD}

Isotopic abundance determination involves fitting synthetic spectra to an observed spectrum. Key ingredients needed for computation of a synthetic spectrum are a suite of appropriate model atmospheres, a line list for TiO, and other contributors, a set of stellar parameters (effective temperature, surface gravity, metallicity, microturbulence $(\xi)$, macroturbulence $(\zeta)$, and projected rotational velocity), and a code for the computation of the synthetic spectra. In this latter context, we use the program MOOG (Sneden 1973) which assumes local thermodynamic equilibrium (LTE) and considers consistently ionization and association of atoms into molecules. In subsequent sections, we describe the employed ingredients.

\subsection{Model Atmospheres}

Model atmospheres were taken from the NEXTGEN grid (Hauschildt et al. 1999) used by Bean et al. (2006) with an interpolation in effective temperature $\left(T_{\text {eff }}\right)$, surface gravity $(\log g)$, and metallicity $([\mathrm{Fe} / \mathrm{H}])$. The NEXTGEN models assume a microturbulence $(\xi)$ of $2 \mathrm{~km} \mathrm{~s}^{-1}$. The adopted composition for the models is a scaled solar composition (i.e., $[\mathrm{X} / \mathrm{Fe}]=0)$ and the solar Fe abundance is taken as $\log \epsilon(\mathrm{Fe})=7.45$.

\footnotetext{
4 IRAF is distributed by the National Optical Astronomy Observatories, which is operated by the Association of Universities for Research in Astronomy, Inc., under cooperative agreement with the National Science Foundation.
} 


\subsection{Line List}

The TiO lines are drawn from Plez (1998) who provides data for the leading electronic transitions: wavelengths, excitation potentials, and $g f$ values. His list includes lines for all five $\mathrm{Ti}$ isotopes in combination with ${ }^{16} \mathrm{O}$. For our primary region (7045$7085 \AA$ ), we imposed a cutoff in strength in order to reject lines that make a negligible contribution to the stellar spectrum. The final list of more than 5500 lines contains in addition to the $0-0$ band $\mathrm{P}, \mathrm{Q}$, and $\mathrm{R}$ branch lines for the five isotopic varieties of $\mathrm{TiO}$, satellite branch lines from the $0-0$ band of ${ }^{48} \mathrm{TiO}$, and ${ }^{48} \mathrm{TiO}$ lines of $\Delta v=+1 \gamma$-system bands. ${ }^{5}$ It will be shown below that, as contributors to the stellar spectra, the satellite branches and the hot bands rank with the lines of the $0-0$ main branch lines from the four less abundant isotopes. A laboratory spectrum of TiO described by Davis et al. (1986) was retrieved from the NSO library. ${ }^{6}$ This spectrum was used to check the wavelengths of the main branch $0-0$ lines for all five isotopic varieties.

The Plez line list includes many more lines than we have included for the computation of synthetic spectra. For inclusion in our line list, lines had to pass a simple test. The strength of each line was estimated from the following relation:

$$
S=\log (\operatorname{ag} f \lambda)-\theta \chi,
$$

where $a$ is the abundance, $g f$ is the $g f$ value, $\chi$ is the excitation energy, and $\theta=5040 / T_{\text {eff }}$. For this estimate, a typical but cool M dwarf temperature of $3300 \mathrm{~K}$ was applied. To test for the significant contributors to the $\mathrm{TiO}$ spectral region, several syntheses were performed, starting with an extremely stringent cutoff, allowing only the strongest lines. This cutoff was reduced by 0.5 dex until the change in the spectra was less than $0.5 \%$. Weaker lines were left out of the syntheses.

The Plez list was also used to provide the $\mathrm{TiO}$ lines, primarily from the $\epsilon$-system's $0-0$ band that contaminate the $8420 \AA$ to $8470 \AA$ interval that provides a determination of the $\mathrm{Ti}$ abundance from Ti I lines; the $0-0$ band's red-degraded head is at $8446 \AA$. For the Ti I lines, we computed solar $g f$ values using the solar flux spectrum (Kurucz et al. 1984), the Kurucz (1993) model solar atmosphere, the microturbulence $\xi=1.13 \mathrm{~km} \mathrm{~s}^{-1}$ (Grupp 2004), and a solar $\mathrm{Ti}$ abundance of $\log \epsilon(\mathrm{Ti})=4.90$ (Asplund et al. 2005).

In all cases, we adopt without alteration, Plez's choices for the $g f$ values based on a combination of laboratory measurements, primarily radiative lifetimes, and quantum chemistry calculations. We also adopt Plez's choice for the TiO dissociation energy of TiO: $D_{0}=6.87_{-0.05}^{+0.07} \mathrm{eV}$, the value determined by experiment (Naulin et al. 1997).

\subsection{Stellar Parameters}

Our principal goal - the determination of the relative abundances of the five Ti isotopes - is, as we show below, insensitive to the adopted effective temperature, gravity, and metallicity of the adopted model atmosphere. A key parameter is the microturbulence because the abundance ratio of a trace isotope to ${ }^{48} \mathrm{Ti}$ is 10 to 20 to 1 or greater and, thus, the ${ }^{48} \mathrm{TiO}$ line may be saturated when its isotopic counterparts are conveniently detectable.

\footnotetext{
5 Lines from $\mathrm{Ti}^{17} \mathrm{O}$ and $\mathrm{Ti}^{18} \mathrm{O}$ are not included in the line list on account of the anticipated low abundances of these isotopes; their abundances are likely less than those of the solar system for which ${ }^{16} \mathrm{O} /{ }^{17} \mathrm{O}=2700$ and ${ }^{16} \mathrm{O} /{ }^{18} \mathrm{O}=$ 480. The complex of stellar TiO lines will include very weak contributions, probably irretrievably blended, from ${ }^{48} \mathrm{Ti}^{18} \mathrm{O}$ for the coolest dwarfs.

6 http://solarch.tuc.noao.edu/diglib/query_by.html
}

Table 2

Stellar Parameters

\begin{tabular}{lccccc}
\hline \hline \multicolumn{1}{c}{ Star } & $\begin{array}{c}T_{\text {eff }} \\
(\mathrm{K})\end{array}$ & $\begin{array}{c}\log g \\
(\mathrm{cgs})\end{array}$ & {$[\mathrm{Fe} / \mathrm{H}]$} & $\begin{array}{c}\xi \\
\left(\mathrm{km} \mathrm{s}^{-1}\right)\end{array}$ & $\begin{array}{c}\zeta \\
\left(\mathrm{km} \mathrm{s}^{-1}\right)\end{array}$ \\
\hline GJ184 & 3700 & 4.7 & -0.5 & 1.0 & 2.0 \\
GJ215 & 3900 & 4.5 & -0.1 & 1.0 & 1.0 \\
GJ378 & 3600 & 4.6 & -0.4 & 1.4 & 0.7 \\
GJ699 & 3134 & 5.1 & -0.8 & 0.6 & 1.4 \\
GJ701 & 3680 & 4.8 & -0.2 & 1.0 & 0.8 \\
GJ725A & 3400 & 4.9 & -0.3 & 1.1 & 1.0 \\
GJ880 & 3640 & 4.7 & 0.0 & 1.0 & 1.5 \\
GJ908 & 3550 & 4.8 & -0.5 & 0.8 & 0.8 \\
LHS178 & 3600 & 5.0 & -1.0 & 1.0 & 1.0 \\
LHS1226 & 3900 & 4.8 & -0.1 & 0.6 & 1.4 \\
LHS2018 & 3750 & 4.7 & -0.5 & 1.0 & 1.0 \\
\hline
\end{tabular}

However, the other stellar parameters are important in determining the metallicity and it is through the variation of the isotopic abundances with metallicity that we test the predictions of GCE. Adopted values for the stellar parameters are given in Table 2 with the discussion in the following subsections providing the adopted methods.

\subsubsection{Effective Temperature}

Initial estimates of $T_{\text {eff }}$ are based on a star's spectral type (Reid et al. 1995) and the spectral type-effective temperature relation provided by Reid \& Hawley (2005). This procedure is anticipated to provide $T_{\text {eff }}$ to about $100 \mathrm{~K}$. Small adjustments to $T_{\text {eff }}$ were made in some cases in order that the Ti abundances from the Ti I and $\mathrm{TiO}$ lines were consistent. For Gl699, we adopt $T_{\text {eff }}$ from Dawson \& DeRobertis (2004) based upon estimates of the star's angular diameter and total flux.

\subsubsection{Surface Gravity}

The $M_{K}$-mass relationship from Delfosse et al. (2000) was exploited as a route to the surface gravity because it has a weak metallicity dependence. This relation with the Bean et al. (2006) $\log g$-mass relation provides an estimate of the surface gravity. Hipparcos parallaxes (Perryman et al. 1997) and Two Micron All Sky Survey (2MASS) $K$ magnitudes (Cutri et al. 2003) are combined to give $M_{K}$. Only LHS178 is not in the Hipparcos catalog and for this star we take the parallax from Gliese \& Jahreiß (1991). Typically, the $\log g$ is estimated to about \pm 0.01 dex but this does not include the systematic errors resulting from the use of the two relationships.

\subsubsection{Microturbulence}

The ideal approach would be to determine $\xi$ from the $\mathrm{TiO}$ spectrum. Across the observed stretch of the $\gamma$-system's $0-0$ band, the ${ }^{48} \mathrm{TiO}$ lines from the $\mathrm{P}, \mathrm{Q}$, and $\mathrm{R}$ lines do not show a sufficient difference in strength to provide a useful estimate of $\xi$. Additionally, many lines are blended with lines of the other isotopic varieties. In principle, one may use the satellite ${ }^{48} \mathrm{TiO}$ lines for the desired comparison of weak and strong lines from which to determine $\xi$. However, the spectrum is such that examples of clean satellite lines are impossible to find; prospective satellite lines are blended with isotopic lines of similar strength from the P, Q, and R lines, and also P, Q, and R lines of the ${ }^{48} \mathrm{TiO} \gamma$-system's hot $(\Delta v=+1)$ bands.

As an alternative to comparing strengths of weak and strong lines of the same molecular or atomic species, we bound $\xi$ from the widths of TiO lines. An upper limit to $\xi$ is determinable from 
imposing syntheses for different $\xi$ on an observed spectrum. After synthetic spectra are convolved with the instrumental profile appropriate for that observation, the upper limits with a slight star-to-star variation run from $1.5 \mathrm{~km} \mathrm{~s}^{-1}$ to $2.5 \mathrm{~km} \mathrm{~s}^{-1}$. These are upper limits because we neglected contributions to the line width from rotation and macroturbulence.

Our assumption regarding macroturbulence is that it equals microturbulence, an empirical result of approximate validity for the Sun and main-sequence stars but taken as an extrapolation for late-K and early-M dwarfs. Rotational velocities of M dwarfs are generally very low (Reiners 2007), say $v \sin i<1 \mathrm{~km} \mathrm{~s}^{-1}$ equivalent to about a $0.2 \mathrm{~km} \mathrm{~s}^{-1}$ apparent contribution to $\xi$. With our assumptions - macroturbulence equals microturbulence and no rotation-the microturbulence for each star is provided. These values of $\xi$ are a factor of $\sqrt{2}$ smaller than the upper limits. The uncertainty is about $\pm 0.5 \mathrm{~km} \mathrm{~s}^{-1}$. Saturation of lines is controlled, of course, by the quadratic combination of the thermal velocity and the microturbulence. The thermal velocity of a TiO molecule at a representative temperature, say $3000 \mathrm{~K}$, is $\sqrt{2 k T / m}=0.9 \mathrm{~km} \mathrm{~s}^{-1}$, and, thus, a microturbulence less than about $1 \mathrm{~km} \mathrm{~s}^{-1}$ has but a small effect on the saturation of lines. In some cases, small adjustments were made to the microturbulence on the basis of fits to the profiles of the Ti I and $\mathrm{TiO}$ lines. The microturbulence is not reliably derivable from the small suite of $\mathrm{Ti}$ I lines used to obtain the $\mathrm{Ti}$ abundance; there are no weak Ti I lines (the molecular haze impedes the measurement of weak lines), and the measured lines cover too limited a range in strength.

Our results for the microturbulence and macroturbulence are completely in line with results from the recent spectroscopic analyses of Woolf \& Wallerstein (2005) and Bean et al. (2006). For the early-M dwarfs, here $T_{\text {eff }}<4000 \mathrm{~K}$, Woolf \& Wallerstein obtained a mean value of $\xi=1.0 \mathrm{~km} \mathrm{~s}^{-1}$ from 19 stars; their estimates are from $\mathrm{Ti}_{\mathrm{I}}$ lines and the usual constraint that the Ti abundance be independent of equivalent width. Bean et al. determined microturbulence $(\xi)$ and macroturbulence $(\eta)$ from a fitting procedure to a suite of atomic line profiles. Their results from five stars gave mean values: $\xi=0.9 \mathrm{~km} \mathrm{~s}^{-1}$ and $\eta=1.0 \mathrm{~km} \mathrm{~s}^{-1}$; note that our assumption $\xi=\eta$ is essentially verified by these results. These $\xi$ determinations from atomic lines are consistent with our measurements reported in Table 2.

\subsubsection{Metallicity}

An estimate of the metallicity is obtained from the Ti I lines, their solar $g f$ values, and the microturbulence values just discussed with an iteration such that the input metallicity for the model atmosphere is equal to that derived from the lines. Four Ti I lines are used in the final determination: $8457.103 \AA$ from multiplet 174 with $\log g f=-1.85,8476.147 \AA$ from multiplet 182 with $\log g f=-1.26$, and $8438.923 \AA$ and $8450.892 \AA$ from multiplet 223 with $\log g f=-0.79$ and -0.84 , respectively. Multiplet numbers are from Moore (1945). The lines in order by strongest to weakest are $8439,8451,8457$, and 8467 $\AA$. Other Ti I lines are in our spectral window but were rejected either because they are seriously blended or are stronger than the above quartet and then sensitive to the (uncertain) damping constants. The $[\mathrm{Fe} / \mathrm{H}]$ is estimated from the $[\mathrm{Ti} / \mathrm{H}]$ using mean trends for the $[\mathrm{Ti} / \mathrm{Fe}]$ versus $[\mathrm{Fe} / \mathrm{H}]$ for thin and thick disk stars (Reddy et al. 2006): $[\mathrm{Ti} / \mathrm{Fe}]=-0.18[\mathrm{Fe} / \mathrm{H}]$ for the thin disk, and $[\mathrm{Ti} / \mathrm{Fe}]=-0.03[\mathrm{Fe} / \mathrm{H}]+0.2$ for thick disk and halo stars.

In determining the $\mathrm{Ti}$ abundance from the $\mathrm{TiO}$ lines, we constrain the analysis by adopting relationships between the $\mathrm{C}, \mathrm{O}$, and $\mathrm{Fe}$ abundances. The $[\mathrm{Ti} / \mathrm{Fe}]$ relation was as above.
Table 3

Metallicity Study

\begin{tabular}{lccccccc}
\hline \hline \multirow{1}{*}{ Star } & \multicolumn{9}{c}{$[\mathrm{Ti} / \mathrm{H}]$} & \multicolumn{5}{c}{ TiO } \\
\cline { 2 - 6 } & \multicolumn{5}{c}{ Ti I } \\
\cline { 2 - 6 } & $8438.9 \AA$ & $8450.9 \AA$ & $8457.1 \AA$ & $8467.1 \AA$ & Mean \\
\hline GJ184 & -0.21 & -0.40 & -0.45 & -0.28 & -0.34 & -0.30 & -0.5 \\
GJ215 & 0.0 & -0.05 & -0.25 & 0.05 & -0.06 & -0.13 & -0.1 \\
GJ378 & -0.09 & -0.19 & -0.34 & -0.11 & -0.18 & -0.28 & -0.4 \\
GJ699 & -0.68 & -0.64 & -0.61 & -0.49 & -0.61 & -0.63 & -0.8 \\
GJ701 & -0.20 & -0.25 & -0.25 & -0.10 & -0.20 & -0.18 & -0.2 \\
GJ725A & -0.18 & -0.23 & -0.21 & -0.04 & -0.17 & -0.30 & -0.3 \\
GJ880 & 0.10 & 0.02 & -0.07 & 0.10 & 0.04 & -0.13 & 0.0 \\
GJ908 & -0.40 & -0.44 & -0.55 & -0.35 & -0.44 & -0.38 & -0.5 \\
LHS178 & -1.00 & -0.95 & -0.95 & -0.75 & -0.97 & -0.55 & -1.0 \\
LHS1226 & -0.12 & -0.22 & -0.20 & -0.06 & -0.10 & -0.05 & -0.1 \\
LHS2018 & -0.36 & -0.46 & -0.50 & -0.35 & -0.37 & -0.39 & -0.5 \\
\hline
\end{tabular}

The $[\mathrm{C} / \mathrm{Fe}]$ and $[\mathrm{O} / \mathrm{Fe}]$ relations, also from Reddy et al. (2006), are: $[\mathrm{C} / \mathrm{Fe}]=-0.23[\mathrm{Fe} / \mathrm{H}]$ for the thin and thick disk and halo stars, and $[\mathrm{O} / \mathrm{Fe}]=-0.19[\mathrm{Fe} / \mathrm{H}]$ for the thin disk and $[\mathrm{O} / \mathrm{Fe}]=-0.25[\mathrm{Fe} / \mathrm{H}]$ for thick disk and halo stars. Adopted solar abundances are $\log \epsilon(\mathrm{C})=8.39$ and $\log \epsilon(\mathrm{O})=8.66$ (Asplund et al. 2005).

These assumptions about the composition are not entirely consistent with the adoption of a scaled solar composition used for the computation of the model atmospheres. This inconsistency will have a negligible effect on the derived $\mathrm{Ti}$ isotopic abundances.

\section{THE METALLICITY OF THE M DWARFS}

The $\mathrm{Ti}$ I lines were each fit using the fitting procedure applied by Bean et al. (2006) assuming the microturbulence and macroturbulence velocities discussed above. Results for the four lines are given in Table 3. This analysis assumes LTE. Results from the four lines are generally consistent: $8467 \AA$, the weakest line, gives an abundance that is about 0.1 dex less than the average, and the $8457 \AA$ line gives an abundance about 0.1 dex more than the average. These small differences have a negligible effect on an interpretation of the trend of isotopic abundances with metallicity. Perhaps, larger effects in the line-to-line scatter and the mean Ti abundance result from our assumption of LTEsee Hauschildt et al. (1997) for a discussion of non-LTE effects on the Ti I spectrum in M dwarfs.

The ${ }^{48} \mathrm{TiO} 0-0$ band lines were fit to obtain a Ti abundance with the results given in Table 3 . The abundance there tabulated includes a correction for the four trace isotopes in order that a direct comparison may be made with the abundance from the atomic lines which necessarily refers to the total $\mathrm{Ti}$ abundance because the isotopic wavelength shifts are negligible.

Agreement between the Ti abundances obtained from atomic and molecular lines is good except for LHS178. In part, this is a result of small adjustments to $T_{\text {eff }}$ (see above) for a few stars: adjustments were $100 \mathrm{~K}$ or less except for LHS2018 where $T_{\text {eff }}$ was lowered by $250 \mathrm{~K}$ from the value indicated by the spectral type. The $[\mathrm{Ti} / \mathrm{H}]$ are converted to $[\mathrm{Fe} / \mathrm{H}]$ by the recipe given above.

The high-resolution spectroscopic model atmosphere (NEXTGEN) analysis of Fe I and Ti $\mathrm{I}$ lines for GJ701 by Woolf \& Wallerstein $(2005)$ gave $[\mathrm{Fe} / \mathrm{H}]=-0.20$ and $[\mathrm{Ti} / \mathrm{H}]=-0.25$ in good agreement with our results (Table 3). For LHS178, our $\mathrm{Ti}$ I lines give $[\mathrm{Ti} / \mathrm{H}]=-0.97$ and $\mathrm{TiO}$ lines $[\mathrm{Ti} / \mathrm{H}]=-0.55$ where the disagreement in part reflects a low quality of fit to 
Table 4

Comparisons with Bonfils et al. (2005) and Casagrande et al. (2008)

\begin{tabular}{lccrrrr}
\hline \hline \multirow{2}{*}{ Star } & \multicolumn{2}{c}{$T_{\text {eff }}$} & & \multicolumn{3}{c}{$[\mathrm{Fe} / \mathrm{H}]$} \\
\cline { 2 - 3 } \cline { 6 - 7 } & CL $^{\mathrm{a}}$ & $\mathrm{CFB}^{\mathrm{b}}$ & & \multicolumn{1}{c}{$\mathrm{B}^{\mathrm{c}}$} & $\mathrm{CFB}^{\mathrm{b}}$ & $\mathrm{CL}^{\mathrm{a}}$ \\
\hline GJ184 & 3700 & 3690 & & -0.3 & -0.2 & -0.5 \\
GJ215 & 3900 & 3950 & & -0.2 & 0.1 & -0.1 \\
GJ378 & 3600 & 3590 & & -0.1 & -0.3 & -0.4 \\
GJ699 & 3134 & 3150 & & -0.3 & -0.6 & -0.8 \\
GJ701 & 3680 & 3560 & & -0.2 & -0.2 & -0.2 \\
GJ725A & 3400 & 3300 & & -0.3 & -0.7 & -0.3 \\
GJ880 & 3640 & 3540 & & 0.0 & 0.0 & 0.0 \\
GJ908 & 3550 & 3560 & & -0.5 & -0.6 & -0.5 \\
LHS178 & 3600 & 3500 & & -0.8 & -1.0 & -1.0 \\
LHS1226 & 3900 & 3700 & & -0.2 & -0.3 & -0.1 \\
LHS2018 & 3750 & 3960 & -0.5 & 0.2 & -0.5 \\
\hline
\end{tabular}

Notes.

a This paper.

b Casagrande et al. (2008).

c Bonfils et al. (2005).

the atomic lines. We adopt the average value which translates to $[\mathrm{Fe} / \mathrm{H}]=-1.0$. The only other estimate of the metallicity of LHS178 is from photometric band strength indices of Gizis (1997). These indices are a measure of $\mathrm{CaH}$ and $\mathrm{TiO}$ band heads. The degeneracy of temperature and abundance can be broken with the double-metal, temperature-sensitive $\mathrm{TiO}$ against the single-metal, less temperature-sensitive molecule of $\mathrm{CaH}$. This technique gave $[\mathrm{Fe} / \mathrm{H}]=-1.0 \pm 0.5$, which agrees with our measurement.

Our determinations of the iron abundance $[\mathrm{Fe} / \mathrm{H}]$ are supported by the photometric calibration of the metallicities of $\mathrm{M}$ dwarfs by Bonfils et al. (2005). Their calibration of $[\mathrm{Fe} / \mathrm{H}]$ in terms of a polynomial expansion involving the absolute magnitude $M_{K}$ and the color index $(V-K)$ was provided by combining two data sets. Spectroscopic $[\mathrm{Fe} / \mathrm{H}]$ abundances obtained by Woolf \& Wallerstein (2005) for late-K and early-M dwarfs provide about half of the calibrators. The other half are dwarfs with spectral types from $\mathrm{K} 7$ to M6 belonging to wide visual binaries in which the primary is a $\mathrm{F}, \mathrm{G}$, or $\mathrm{K}$ dwarf whose spectrum is amenable to an abundance analysis by standard spectroscopic techniques. The cool secondary is assumed-reasonably so-to have the $[\mathrm{Fe} / \mathrm{H}]$ of its primary companion. These 48 calibrators provide the polynomial expansion. ${ }^{7}$ Table 4 and Figure 1 show the comparison between our results and the values given by the Bonfils et al.'s calibration. The agreement is satisfactory. A possibly discrepant point is that for GJ699 but for this star the $M_{K}$ is outside the limits of the calibration.

A photometric technique for the determination of $T_{\text {eff }}$ and metallicity has been developed by Casagrande et al. (2008) using Johnson-Cousins and 2MASS near-infrared photometry. L. Casagrande (2008, private communication) has kindly applied their technique to our stellar sample. A comparison with our results is given in Table 4 and Figure 2. The effective temperatures obtained by Casagrande et al. agree within $100 \mathrm{~K}$ with ours except for LHS2018 and LHS1226, a result generally consistent with the expected errors of the two techniques. Interpreting the photometric metallicity $[\mathrm{M} / \mathrm{H}]$ as $[\mathrm{Fe} / \mathrm{H}]$, these results agree quite well with ours. Casagrande et al. note agreement between their $[\mathrm{M} / \mathrm{H}]$ and recent determinations at the 0.2 dex level

\footnotetext{
7 Johnson \& Apps (2009) remark that the photometric calibration underestimated $[\mathrm{Fe} / \mathrm{H}]$ (relative to spectroscopic estimates) for metal-rich $\mathrm{M}$ dwarfs. This effect is unimportant here; our sample does not include metal-rich
} $([\mathrm{Fe} / \mathrm{H}]>0)$ stars.

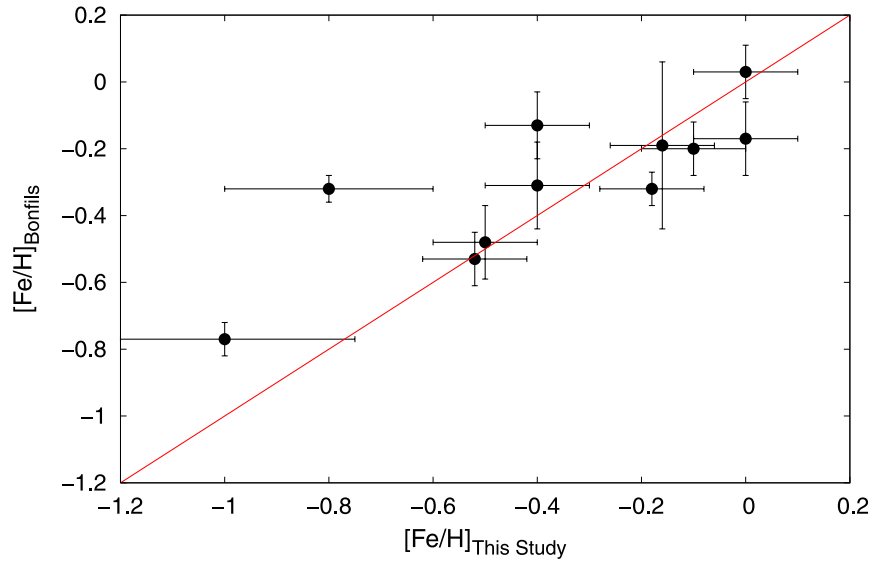

Figure 1. Comparison between our spectroscopic $[\mathrm{Fe} / \mathrm{H}]$ and metallicities derived from the Bonfils et al. (2005) photometric calibration (their Equation (1)). The red line indicates exact equality between the results from the two methods.

(A color version of this figure is available in the online journal.)

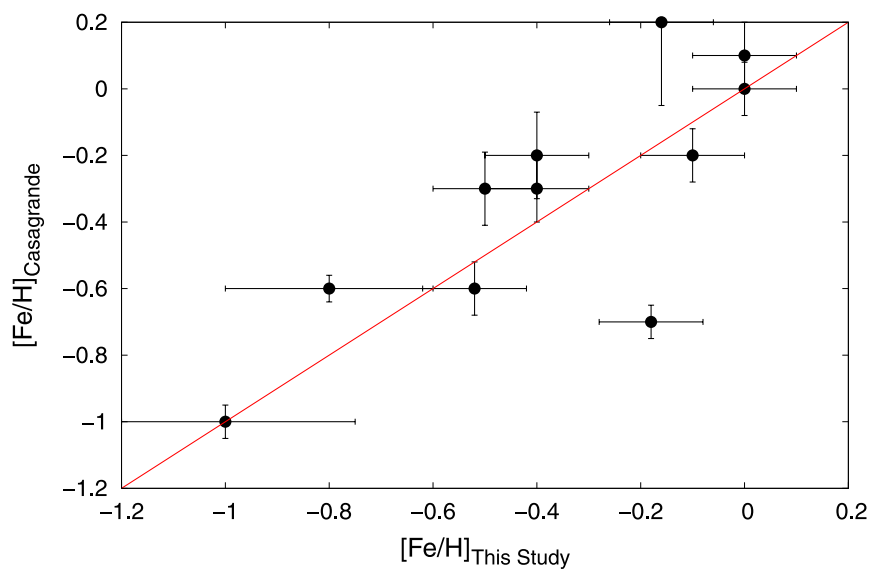

Figure 2. Comparison between our spectroscopic $[\mathrm{Fe} / \mathrm{H}]$ and metallicities derived from the Casagrande et al. (2008) photometric calibration (L. Casagrande 2008 , private communication). The red line indicates exact equality between the results from the two methods.

(A color version of this figure is available in the online journal.)

in general. Eight of our 11 stars match the photometric metallicity to within \pm 0.2 dex. The exceptions show differences (Us - Casagrande) of $-0.3,+0.4$, and -0.7 dex where only the latter for LHS2018 might be considered a concern. For each of these three, the Bonfils et al. (2005) recipe gives results in good agreement with ours.

In summary, the $[\mathrm{Fe} / \mathrm{H}]$ in Table 3 appear reliable at the \pm 0.2 dex level. These values define well the $x$-axis in the plots of isotopic ratios versus metallicity that are used to test predictions of GCE.

\section{THE ISOTOPIC ABUNDANCES}

Isotopic abundance ratios are derived by fitting synthetic spectra to an observed spectrum. Ratios of the four lesser abundant isotopes with respect to ${ }^{48} \mathrm{Ti}$ are sensitive primarily to the microturbulence. Ratios among the four lesser abundant isotopes are insensitive to the microturbulence. Both forms for expressing the isotopic ratios are sensitive to blends chiefly from weak ${ }^{48} \mathrm{TiO}$ lines including the satellite transitions from the same $0-0$ band that provides the main lines of interest and from hot bands of the $\gamma$ system. In the following subsections, we discuss the determinations of isotopic ratios. 

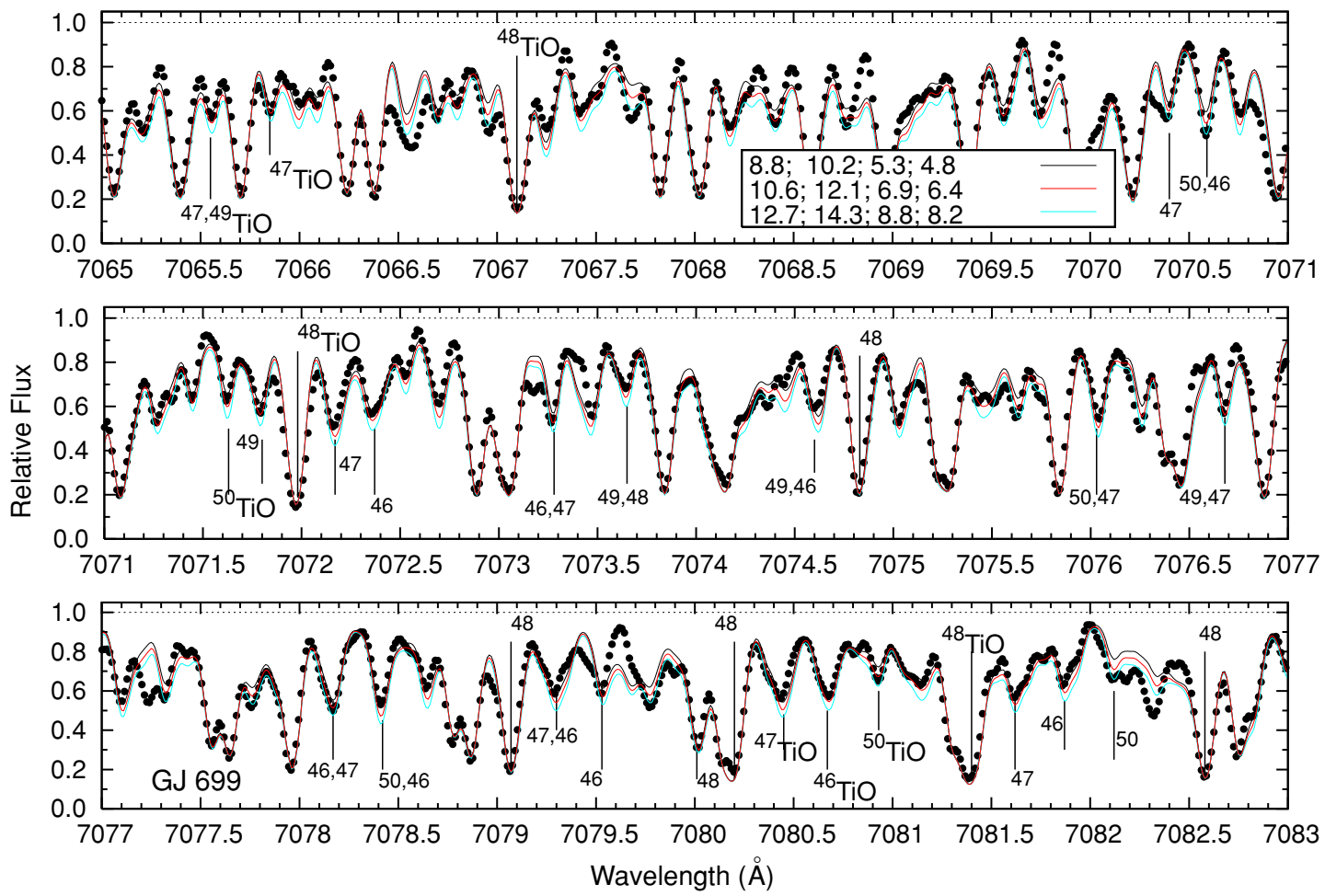

Figure 3. Spectrum of GJ699 for $7065 \AA$ to $7083 \AA$ showing absorption lines for all five varieties of ${ }^{i}$ TiO from the $0-0$ band of the $\gamma$ system. The strongest lines are from ${ }^{48} \mathrm{TiO}$. Weaker lines include lines from the other Ti isotopes with several key lines and blends labeled by the mass number. Synthetic spectra for three isotopic mixes are shown with the key in the upper panel. All three synthetic spectra fit the ${ }^{48} \mathrm{TiO}$ lines. The key to the isotopic mixes is given on the figure with left to right the abundances of ${ }^{46} \mathrm{Ti},{ }^{47} \mathrm{Ti},{ }^{49} \mathrm{Ti}$, and ${ }^{50} \mathrm{Ti}$ expressed in percent relative to the ${ }^{48} \mathrm{Ti}$ abundance.
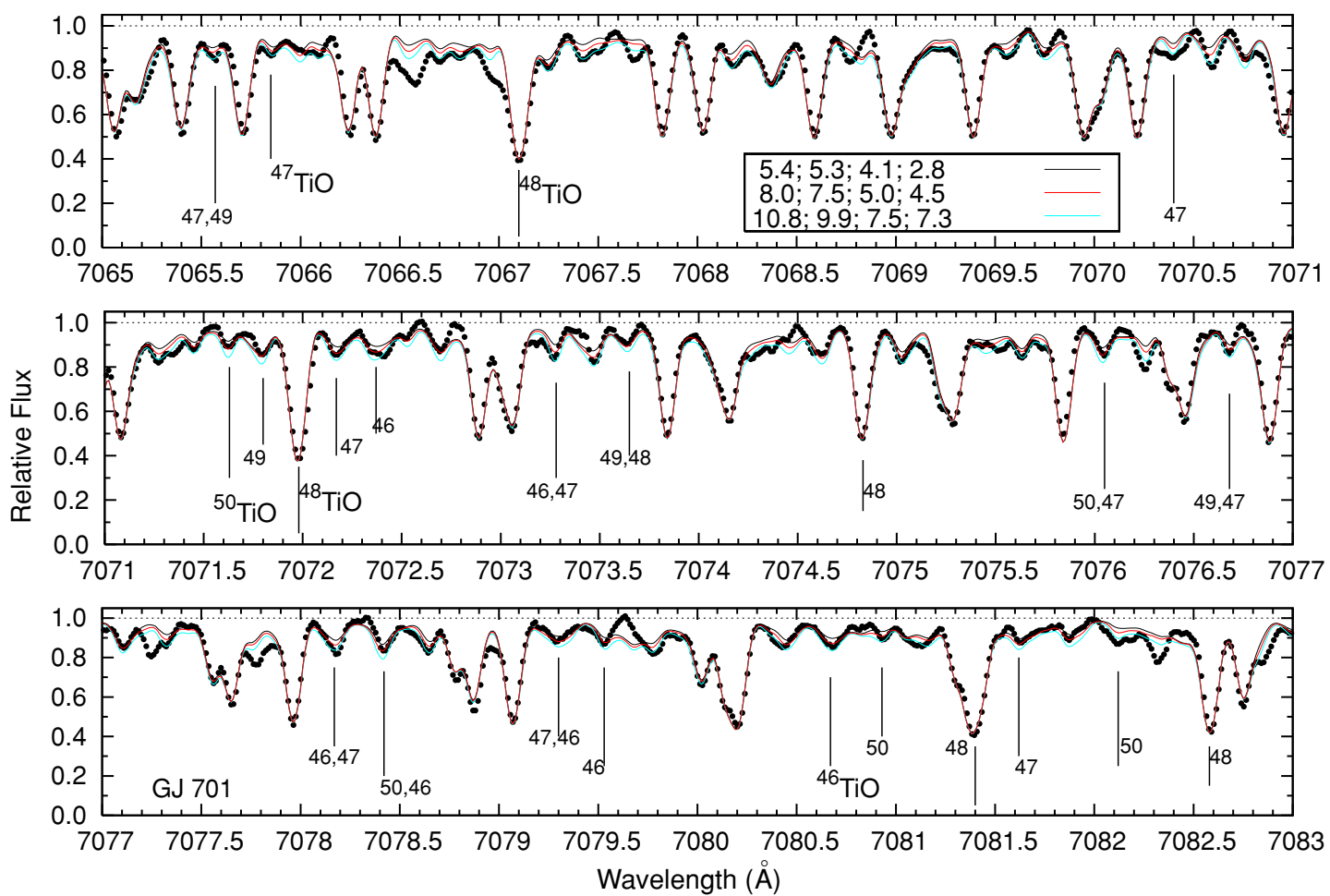

Figure 4. Spectrum of GJ701 for $7065 \AA$ to $7083 \AA$ showing absorption lines for all five varieties of ${ }^{i}$ TiO from the $0-0$ band of the $\gamma$ system. The strongest lines are from ${ }^{48} \mathrm{TiO}$. Weaker lines are from the other Ti isotopes with several key lines and blends labeled by the mass number. Synthetic spectra for three isotopic mixes are shown with the key in the upper panel. All three synthetic spectra fit the ${ }^{48} \mathrm{TiO}$ lines. The key to the isotopic mixes is given on the figure with left to right the abundances of ${ }^{46} \mathrm{Ti},{ }^{47} \mathrm{Ti},{ }^{49} \mathrm{Ti}$, and ${ }^{50} \mathrm{Ti}$ expressed in percent relative to the ${ }^{48} \mathrm{Ti}$ abundance. 

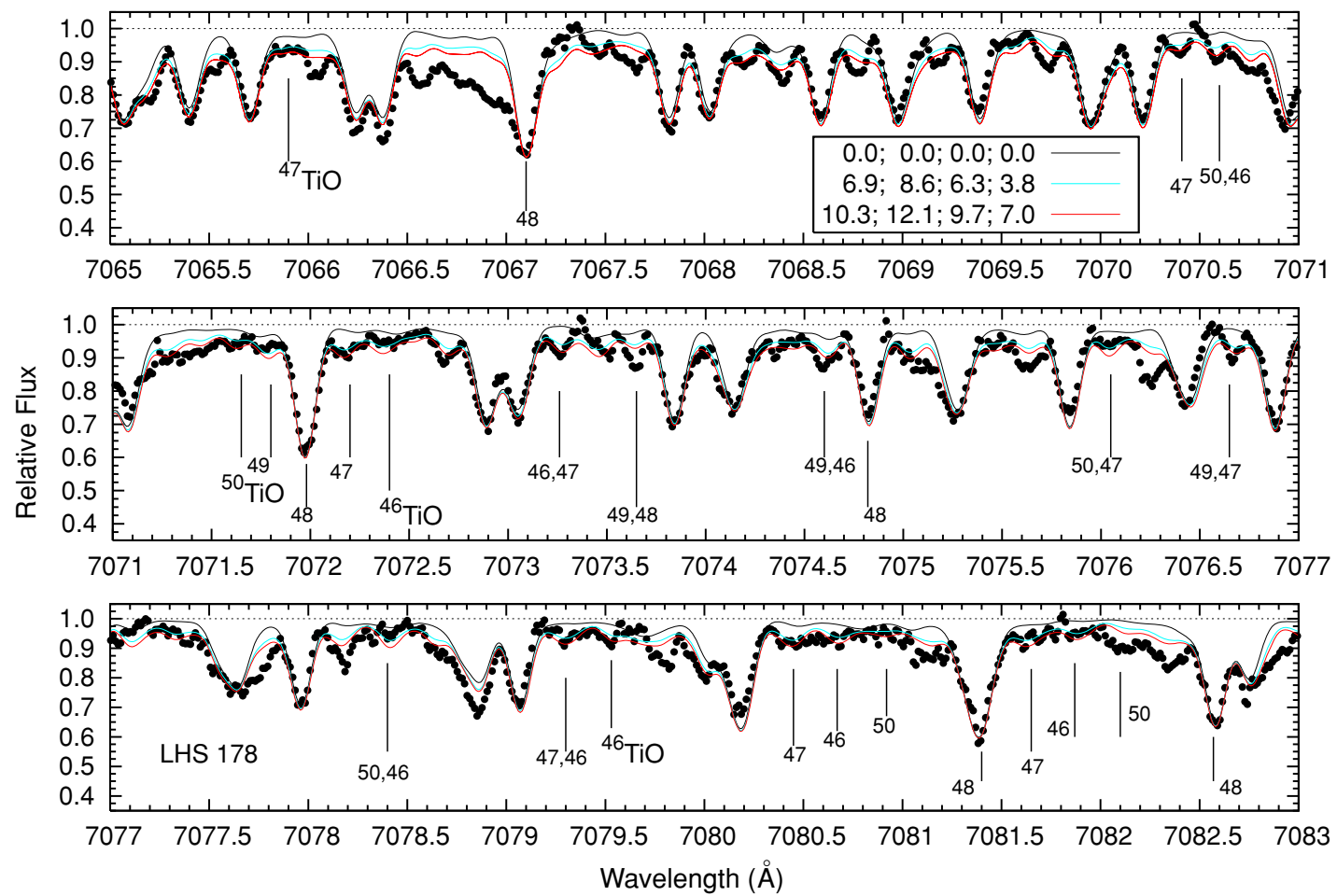

Figure 5. Spectrum of LHS178 for $7065 \AA$ to $7083 \AA$ A showing absorption lines for all five varieties of ${ }^{i} \mathrm{TiO}$ from the $0-0$ band of the $\gamma$ system. The strongest lines are from ${ }^{48} \mathrm{TiO}$. Weaker lines are from the other Ti isotopes with several key lines and blends labeled by the mass number. Synthetic spectra for three isotopic mixes are shown with the key in the upper panel. All three synthetic spectra fit the ${ }^{48} \mathrm{TiO}$ lines. The key to the isotopic mixes is given on the figure with left to right the abundances of ${ }^{46} \mathrm{Ti},{ }^{47} \mathrm{Ti},{ }^{49} \mathrm{Ti}$, and ${ }^{50} \mathrm{Ti}$ expressed in percent relative to the ${ }^{48} \mathrm{Ti}$ abundance. The synthetic spectrum for pure ${ }^{48} \mathrm{TiO}$ shows that the collective contribution from the four lesser abundant $\mathrm{Ti}$ isotopes is present here.
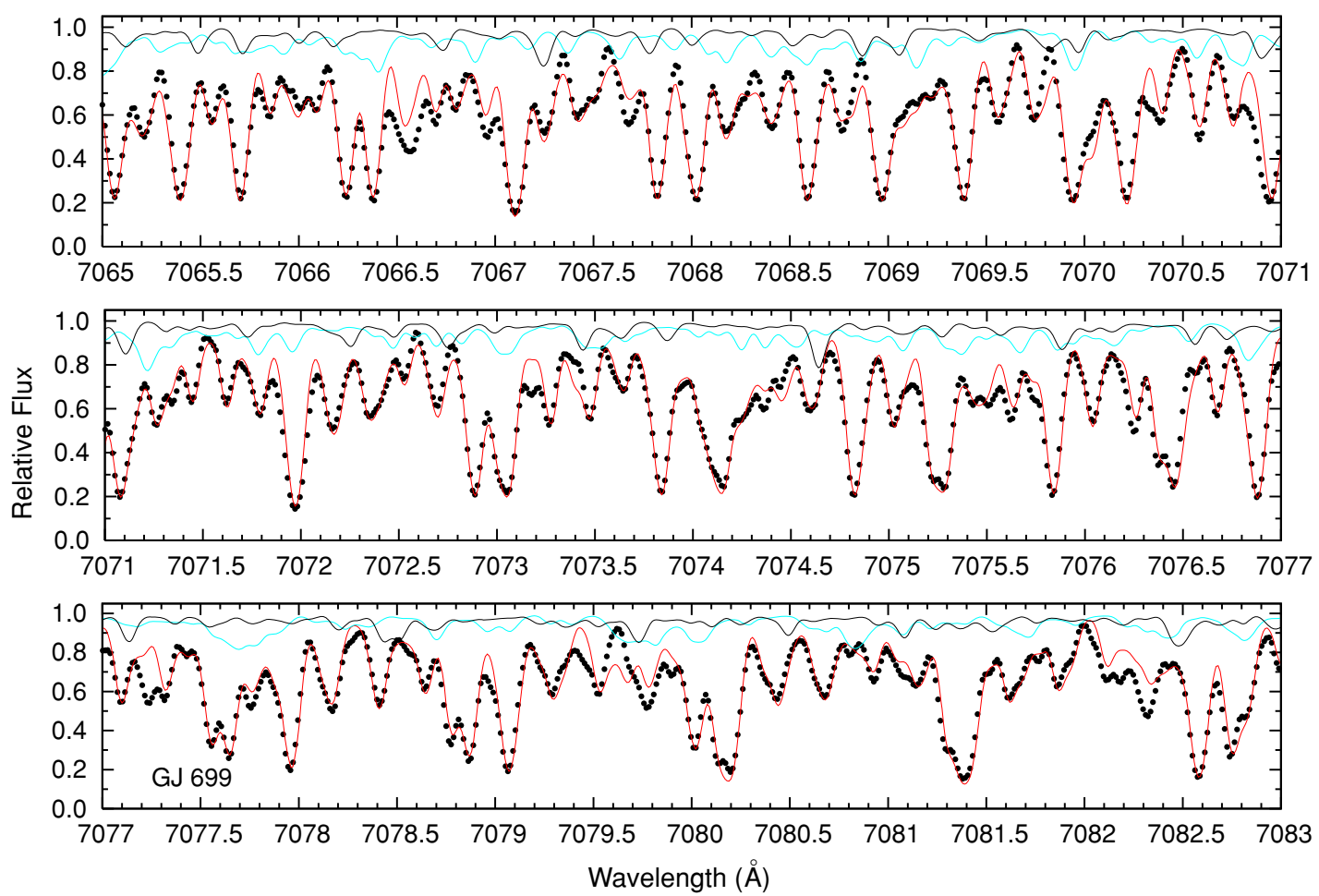

Figure 6. Observed spectrum of GJ699 for $7075 \AA$ to $7083 \AA$ Aith synthetic spectra for three different selections of TiO lines: (1) lines (all Ti isotopes) from the P, Q, and $\mathrm{R}$ branches of the $0-0$ band of the $\mathrm{TiO} \gamma$ system (red line), (2) satellite lines of the $0-0$ band (light blue line), and (3) lines of the $\Delta v=+1$ bands of the $\gamma$ system (black line).

\subsection{Observed and Synthetic Spectra: General Remarks}

The strength of the $\mathrm{TiO}$ lines changes appreciably, as expected, across the stellar sample. These lines are strongest in
GJ699 and weakest in GJ215. Lines from the four trace isotopes are prominent in stars with strong $\mathrm{TiO}$ yet clearly present in sample stars with weak TiO. Isotopic abundances are determined 
from matching synthetic to observed spectra. Figures 3-5 show these spectra for representative stars: GJ699 with strong TiO, GJ701 with medium strength TiO, and LHS178 with weaker $\mathrm{TiO}$ (and our lowest metallicity star).

In Figure 3 for GJ699, the abundance of ${ }^{48} \mathrm{Ti}$ is fixed and synthetic spectra for three different isotopic fractions ${ }^{i} \mathrm{Ti} /{ }^{48} \mathrm{Ti}$ are shown. By inspection, one can identify the features to which one or more of the trace isotopes contributes. The measurement of isotopic fractions is discussed below. Inspection also suffices to show wavelength regions for which the synthetic spectra fail to match the observed spectrum. In those cases where the observed spectrum shows the greater absorption, one presumes that unidentified lines not included in the line list used for computing the synthetic spectrum are depressing the spectrum. These regions are ignored in assessing the isotopic fractions. Surprisingly, there are places where the synthetic spectra shows more absorption than the observed spectrum.

The TiO molecule's contribution to the observed spectra is not simply from the $\gamma$-system's $0-0$ band and its $\mathrm{P}, \mathrm{Q}$, and $\mathrm{R}$ lines and their five isotopic components. The $0-0$ band satellite branches contribute lines. In addition, the $\gamma$-system's $\Delta v=+1$ sequence contributes lines. Our inclusion of satellite and $\Delta v=+1$ lines adds "noise" to the spectrum. By way of illustration, we show in Figure 6 for GJ699 the separate contributions to the synthetic spectrum of (1) the $\mathrm{TiO} 0-0$ band $\mathrm{P}, \mathrm{Q}$, and R lines from all isotopes, (2) the $0-0$ band satellite ${ }^{48} \mathrm{TiO}$ lines, and ( 3$)^{48} \mathrm{TiO}$ lines from the $\Delta v=+1$ bands. It is obvious that contributions (2) and (3) are comparable to that from the four trace isotopes in (1). Strengths and positions of the satellite lines are most probably reliably represented in Plez's line list. Some of the $\Delta v=+1$ lines have not been recorded on laboratory spectra and their predicted wavelengths use molecular constants beyond the range in which they have been established. In addition, their $g f$ values are subject to greater uncertainty than are the values for the $0-0$ band. It is because of these two latter uncertainties that the $\Delta v=+1$ lines contribute unwanted noise. Our list contains only about six atomic lines and these we do not identify separately.

No synthetic spectrum is ever a perfect fit to an observed spectrum. Our synthetic spectra are no exception. Failures of the synthetic spectra may be put in two classes: (1) the observed spectrum is stronger than the synthetic spectrum, and (2) and the reverse of this where the synthetic spectrum shows absorption stronger than in observed. In principle, both (1) and (2) are open to simple and obvious interpretations. As noted above, (1) admits of the possibility that the adopted line list is missing lines or the strengths of included lines are underestimated (i.e., adopted $g f$ values are too small). Similarly, (2) may have a simple explanation, i.e., the adopted $g f$ values are too large.

The impossibility or indeed the inevitability of composing a thoroughly complete line list means that one must accept in any comparison occurrences of class (1) and (2) failures. Given the very high quality fit of synthetic to observed spectra, as demonstrated in Figures 3, 4, and 5, a low frequency of failures cannot surely adversely affect determinations of the isotopic ratios. Our impression is that wavelength errors for the lines from the $\Delta v=+1$ bands may be largely responsible for the failures of class (1) and (2). There are, however, places where the synthetic spectrum with just the $0-0$ main lines (all five isotopes) is stronger than the observed spectrum. Two such examples are seen in Figure 6 at $7074.45 \AA$ and $7079.65 \AA$. Scrutiny of the line list and the laboratory spectrum of TiO shows that the $\mathrm{TiO}$ lines from the less abundant isotopes are at
Table 5

Primary TiO Lines

\begin{tabular}{|c|c|c|}
\hline$\lambda(\AA)$ & Transition & Isotopes \\
\hline 7058.7 & $\mathrm{R}_{3}(33)$ & 48 \\
\hline 7060.4 & $\mathrm{Q}_{3}(10) \mathrm{R}_{3}(36)$ & 48 \\
\hline 7062.5 & $\mathrm{Q}_{3}(15) \mathrm{R}_{3}(39)$ & 47 \\
\hline 7065.9 & $\mathrm{Q}_{3}(21)$ & 47 \\
\hline 7067.1 & $\mathrm{P}_{3}(12) \mathrm{Q}_{3}(23) \mathrm{R}_{3}(45)$ & 48 \\
\hline 7070.4 & $\mathrm{Q}_{3}(27)$ & 47 \\
\hline 7070.6 & $\mathrm{Q}_{3}(27)$ & 46,50 \\
\hline 7071.6 & $\mathrm{Q}_{3}(29) \mathrm{P}_{3}(17) \mathrm{R}_{3}(50)$ & 50 \\
\hline 7071.8 & $\mathrm{Q}_{3}(29) \mathrm{P}_{3}(17) \mathrm{R}_{3}(50)$ & 49 \\
\hline 7072.0 & $\mathrm{Q}_{3}(29) \mathrm{P}_{3}(17) \mathrm{R}_{3}(50)$ & 48 \\
\hline 7072.4 & $Q_{3}(29) P_{3}(17) R_{3}(50)$ & 46 \\
\hline 7072.2 & $\mathrm{Q}_{3}(29) \mathrm{P}_{3}(17) \mathrm{R}_{3}(50)$ & 47 \\
\hline 7073.3 & $\mathrm{Q}_{3}(30) \mathrm{P}_{3}(18) \mathrm{R}_{3}(51)$ & 46,47 \\
\hline 7073.7 & $\mathrm{Q}_{3}(31)$ & 49 \\
\hline 7074.9 & $\mathrm{Q}_{3}(32)$ & 48 \\
\hline 7076.1 & $\mathrm{Q}_{3}(33) \mathrm{P}_{3}(21) \mathrm{R}_{3}(54)$ & 47,50 \\
\hline 7076.7 & $\mathrm{Q}_{3}(34) \mathrm{R}_{3}(54)$ & 47,49 \\
\hline 7078.2 & $\mathrm{Q}_{3}(35) \mathrm{R}_{3}(55)$ & 46,47 \\
\hline 7078.4 & $\mathrm{Q}_{3}(35) \mathrm{P}_{3}(23) \mathrm{R}_{3}(56)$ & 46,50 \\
\hline 7079.3 & $\mathrm{Q}_{3}(36) \mathrm{P}_{3}(23) \mathrm{R}_{3}(56)$ & 46,47 \\
\hline 7079.5 & $\mathrm{Q}_{3}(36)$ & 46 \\
\hline 7080.5 & $\mathrm{Q}_{3}(37) \mathrm{R}_{3}(57)$ & 47 \\
\hline 7080.7 & $\mathrm{Q}_{3}(37) \mathrm{R}_{3}(57)$ & 46 \\
\hline 7080.9 & $\mathrm{Q}_{3}(38) \mathrm{P}_{3}(25) \mathrm{R}_{3}(58)$ & 50 \\
\hline 7081.4 & $\mathrm{Q}_{3}(38) \mathrm{P}_{3}(25) \mathrm{R}_{3}(58)$ & 48 \\
\hline 7081.7 & $\mathrm{Q}_{3}(38) \mathrm{P}_{3}(25) \mathrm{R}_{3}(58)$ & 47 \\
\hline 7081.9 & $\mathrm{Q}_{3}(38) \mathrm{P}_{3}(25) \mathrm{R}_{3}(58)$ & 46 \\
\hline 7082.6 & $\mathrm{Q}_{3}(39) \mathrm{P}_{3}(26)$ & 48 \\
\hline 7083.2 & $\mathrm{Q}_{3}(39) \mathrm{P}_{3}(26) \mathrm{R}_{3}(59)$ & 46,47 \\
\hline 7083.4 & $\mathrm{Q}_{3}(40)$ & 50 \\
\hline
\end{tabular}

their measured wavelengths and there are no atomic lines in the line list that are contributing unwanted absorption. Furthermore, these discrepancies are seen across the sample at the same (stellar) wavelengths and are not, therefore, attributable to noise, incorrect correction for telluric $\left(\mathrm{H}_{2} \mathrm{O}\right)$ absorption lines or emission $(\mathrm{OH})$ from the night sky. One may wonder if these discrepancies arise from stellar emission lines.

\subsection{The Isotopic Fractions}

In the final fitting of synthetic spectra to an observed spectrum, the microturbulence and macroturbulence are held fixed. A determination of the ${ }^{48} \mathrm{Ti}$ abundance from a fit to the leastblended ${ }^{48} \mathrm{TiO}$ features is made. Below, we discuss the uncertainty in this abundance arising, principally, from the influence of the microturbulence on the saturation of these lines. Next, the isotopic fractions are determined.

An automated fitting routine was adapted for determining these fractions. For the final determinations, an inspection of the fit between the observed spectrum and synthetic spectra for several different isotopic mixtures was made of selected features. The selection (Table 5) emphasizes those features to which one or just two isotopes contribute. For the lesser abundant isotopes, we started with the ${ }^{47} \mathrm{TiO}$-dominated features of which there were several. For each an abundance for ${ }^{47} \mathrm{TiO}$ was determined and a weight assigned from the quality of the best fit. The weighted average abundance of ${ }^{47} \mathrm{TiO}$ and its dispersion was calculated and then adopted for the analysis of blends to which ${ }^{47} \mathrm{TiO}$ is a contributor. Next, we considered ${ }^{46} \mathrm{TiO}$ features in the same manner. Finally, ${ }^{49} \mathrm{TiO}$ and ${ }^{50} \mathrm{TiO}$ were considered. Isotope ratios are simply obtained from these molecular abun- 
Table 6

${ }^{i} \mathrm{TiO}$ Abundances

\begin{tabular}{|c|c|c|c|c|}
\hline \multirow[t]{3}{*}{$\lambda(\AA)$} & \multirow[t]{3}{*}{ Isotope } & \multicolumn{3}{|c|}{ Star } \\
\hline & & GJ699 & GJ701 & LHS178 \\
\hline & & $\log \epsilon\left({ }^{i} \mathrm{Ti}\right)$ & $\log \epsilon\left({ }^{i} \mathrm{Ti}\right)$ & $\log \epsilon\left({ }^{i} \mathrm{Ti}\right)$ \\
\hline 7072.4 & 46 & 3.18 & 3.64 & 3.11 \\
\hline 7079.5 & 46 & 3.21 & 3.70 & $\ldots$ \\
\hline 7080.7 & 46 & 3.18 & 3.70 & 3.11 \\
\hline 7081.9 & 46 & 3.17 & $\ldots$ & 3.21 \\
\hline 7073.3 & $46[47]^{\mathrm{a}}$ & 3.29 & 3.76 & $\ldots$ \\
\hline 7078.2 & $46[47]$ & 3.26 & 3.76 & $\ldots$ \\
\hline 7079.3 & $46[47]$ & 3.18 & 3.46 & 3.11 \\
\hline \multirow[t]{2}{*}{7083.2} & $46[47]$ & 3.23 & 3.64 & $\cdots$ \\
\hline & Mean $\left({ }^{46} \mathrm{Ti}\right)$ & $3.20 \pm 0.1$ & $3.62 \pm 0.06$ & $3.35 \pm 0.09$ \\
\hline 7062.5 & 47 & 3.21 & 3.46 & 3.35 \\
\hline 7065.9 & 47 & 3.31 & 3.70 & 3.35 \\
\hline 7070.4 & 47 & 3.35 & 3.85 & 3.51 \\
\hline 7072.2 & 47 & 3.21 & 3.64 & 3.44 \\
\hline 7080.5 & 47 & 3.21 & $\ldots$ & 3.44 \\
\hline \multirow[t]{2}{*}{7081.7} & 47 & 3.21 & 3.56 & 3.44 \\
\hline & Mean $\left({ }^{47} \mathrm{Ti}\right)$ & $3.25 \pm 0.1$ & $3.77 \pm 0.07$ & $3.43 \pm 0.07$ \\
\hline 7065.6 & $49[47]$ & 2.99 & 3.56 & $\ldots$ \\
\hline 7071.8 & 49 & 3.03 & 3.46 & 3.15 \\
\hline 7073.7 & 49 & 2.99 & 3.34 & $\ldots$ \\
\hline 7074.6 & $49[46]$ & $\ldots$ & $\ldots$ & 3.32 \\
\hline \multirow[t]{2}{*}{7076.7} & $49[47]$ & 3.03 & 3.56 & 3.40 \\
\hline & Mean $\left({ }^{49} \mathrm{Ti}\right)$ & $3.01 \pm 0.08$ & $3.44 \pm 0.11$ & $3.34 \pm 0.1$ \\
\hline 7071.6 & 50 & 2.95 & 3.37 & 3.10 \\
\hline 7080.9 & 50 & 3.04 & 3.53 & 3.20 \\
\hline 7083.4 & 50 & 2.90 & 3.43 & 3.20 \\
\hline 7076.1 & $50[47]$ & 2.84 & 3.43 & $\ldots$ \\
\hline 7078.4 & $50[46]$ & 2.69 & 3.43 & $\ldots$ \\
\hline \multirow[t]{2}{*}{7070.6} & $50[46]$ & 3.23 & $\ldots$ & 3.29 \\
\hline & Mean $\left({ }^{50} \mathrm{Ti}\right)$ & $2.98 \pm 0.1$ & $3.42 \pm 0.11$ & $3.19 \pm 0.15$ \\
\hline 7058.7 & 48 & 4.23 & 4.74 & 4.38 \\
\hline 7060.4 & 48 & 4.18 & 4.74 & 4.41 \\
\hline 7067.1 & 48 & 4.12 & 4.74 & 4.34 \\
\hline 7072.0 & 48 & 4.23 & 4.74 & 4.31 \\
\hline 7074.9 & 48 & 4.18 & 4.74 & 4.27 \\
\hline 7081.4 & 48 & 4.08 & 4.74 & 4.38 \\
\hline \multirow[t]{2}{*}{7082.6} & 48 & 4.14 & 4.74 & 4.35 \\
\hline & Mean $\left({ }^{48} \mathrm{Ti}\right)$ & $4.17 \pm 0.16$ & $4.74 \pm 0.03$ & $4.35 \pm 0.1$ \\
\hline
\end{tabular}

Note.

a The isotope in square brackets is given its mean abundance in estimating the abundance of the other isotope.

dances. An inspection of the complete wavelength region was conducted to check for the quality of the fit with the synthetic spectrum computed for the final isotopic abundances.

Table 6 illustrates this procedure for the three representative stars: GJ699, GJ701, and LHS178. The feature-to-feature scatter in the abundance of a given trace isotope reflects partly the noise in the observed spectrum and partly the complexity of the spectrum and in particular contributions from satellite and $\Delta v=+1$ lines. Table 7 summarizes the Ti isotopic abundances for each of the program stars. In Table 8, the isotopic abundances are expressed as a fraction of the total (all isotopes) Ti. The uncertainties in Tables 7 and 8 calculated from the line-to-line scatter are one contributor to the total uncertainty; systematic errors are discussed in the following section.

LHS178 obviously enjoys a special place in the interpretation of the GCE predictions; it is the most metal-poor star and the sole representative of the Galactic halo in our small sample.
It was, as noted above, the only star observed at a resolving power of 60,000 and not 120,000 . Figure 5 shows three synthetic spectra and the observed spectrum. The spectrum for pure ${ }^{48} \mathrm{TiO}$ including satellite lines and lines from the $\Delta v=+1$ bands does not fit the observed spectrum, and, therefore, it is difficult to escape the conclusion that the less abundant $\mathrm{Ti}$ isotopes have left their imprint on the observed spectrum. A comparison of observed spectra for other stars with $\mathrm{TiO}$ strengths similar to those for LHS178 suggests too that the less abundant isotopes are contributors to the LHS178 spectrum. Nonetheless, their abundances are plotted as upper limits in Figures 7 and 8.

\subsection{Systematic Errors}

The relative abundances of trace isotopes are only very weakly dependent on the adopted stellar parameters. This is not the case for the ratios with respect to ${ }^{48} \mathrm{Ti}$ because the ${ }^{48} \mathrm{TiO}$ lines are saturated, quite severely so for stars like GJ699 and less severely so for GJ701; the ratio of the depths of the ${ }^{48} \mathrm{TiO}$ to ${ }^{47} \mathrm{TiO}$ lines is smaller than the approximately 12 to 1 ratio of the abundances. Saturation brings into prominence the sensitivity of the measured isotopic fractions (abundances relative to ${ }^{48} \mathrm{Ti}$ ) to the microturbulence.

Errors introduced to the isotopic ratios by errors in the atmospheric parameters were characterized through a series of syntheses starting with a synthetic spectrum for the standard atmospheric parameters with $1 \%$ noise added. Three different stars were represented: a cool star much like GJ699, a medium temperature star like GJ701, and a warm star mimicking the TiO-weak stars. With these as the "observed" spectra, the $\chi^{2}$ minimization routine found the best-fit isotopic abundances as each of the parameters was changed. Adjustments to $T_{\text {eff }}$ of $\pm 100 \mathrm{~K}$ and to $\log g$ of \pm 0.5 dex resulted in nearly constant changes to the abundances of each of the varieties ${ }^{i} \mathrm{TiO}$ with the result that the isotopic abundance changes were less than about $1 \%-2 \%$, changes less than the errors arising from the line-to-line scatter. The abundance changes were approximately \pm 0.2 dex for the temperature change and \pm 0.09 dex for the gravity change. An $[\mathrm{Fe} / \mathrm{H}]$ change of \pm 0.2 dex in the adopted value for NEXTGEN model atmosphere introduces a small change in isotopic abundances and negligible changes in the isotopic fractions.

Adjustments to the adopted microturbulence $\xi$ from an assumed value of $1 \mathrm{~km} \mathrm{~s}^{-1}$ and across the uncertainty range of $\pm 0.5 \mathrm{~km} \mathrm{~s}^{-1}$ provide small changes in the isotopic fractions when referenced to the ${ }^{48} \mathrm{Ti}$ or total $\mathrm{Ti}$ abundance for all stars except those with the strongest TiO lines (e.g., GJ699). For GJ701, for example, the ${ }^{48} \mathrm{TiO}$ abundance was decreased by about 0.03 dex for the increase in $\xi$ from 1.0 to $1.5 \mathrm{~km} \mathrm{~s}^{-1}$ with isotopic abundance ratios relative to ${ }^{48} \mathrm{Ti}$ changing by $0.02 \mathrm{dex}$ or less. The $\xi$ increase has a greater effect for the GJ699-like stars with the ${ }^{48} \mathrm{TiO}$ abundance decreasing by 0.10 dex and the isotopic ratios relative to ${ }^{48} \mathrm{Ti}$ increasing by about $0.06 \mathrm{dex}$. These changes are of opposite sign and smaller for the reduction in $\xi$ by $0.5 \mathrm{~km} \mathrm{~s}^{-1}$ because of the contribution of the thermal velocities to the total velocity controlling the saturation of the lines. These systematic uncertainties arising from $\xi$ are no larger, even smaller than, the random errors from the fitting of the synthetic spectra and the blending arising from the satellite and $\Delta v=+1$ lines. Certainly, the ratios among the four lesser abundant isotopes are dominated by random and not systematic errors. 


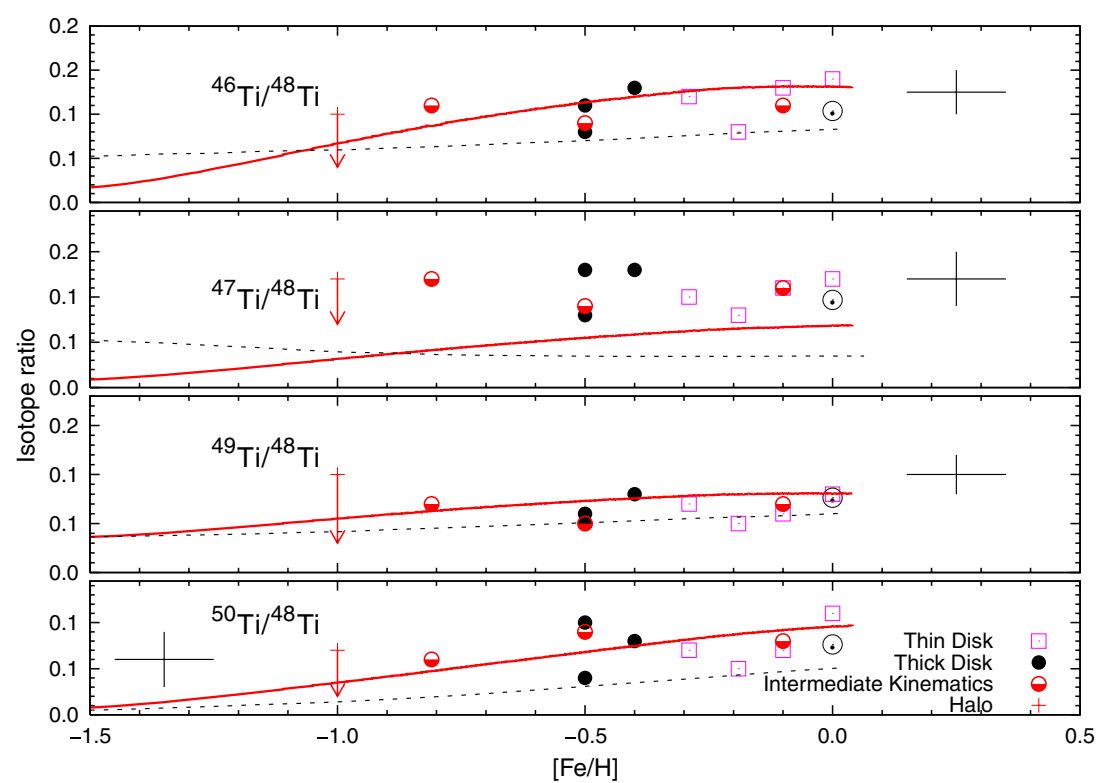

Figure 7. Observed and predicted isotopic ratios ${ }^{i} \mathrm{Ti} /{ }^{48} \mathrm{Ti}$ over the $[\mathrm{Fe} / \mathrm{H}]$ range 0 to -1.5 . Stellar populations of our stars are represented as follows: thick disk $=$ filled circles, thin disk $=$ unfilled squares, halo $=$ cross (arrow indicates an upper limit), and thick-thin disk $=$ intermediate kinematics $=$ half-filled circle. Predictions are from N. Prantzos (2008, private communication; red line) and C. Kobayashi (2008, private communication; black dashed line). An indication of representative uncertainty is given by the cross in each panel. Solar system ratios are denoted at $[\mathrm{Fe} / \mathrm{H}]=0$ by the standard symbol for the Sun.

(A color version of this figure is available in the online journal.)

Table 7

Isotopic Ti Abundances

\begin{tabular}{lcccccc}
\hline \hline \multirow{2}{*}{ Star } & \multicolumn{5}{c}{$\log \epsilon\left({ }^{i} \mathrm{Ti}\right)$} \\
\cline { 2 - 7 } & $i=46$ & 47 & 48 & 49 & 50 & Total \\
\hline GJ184 & $3.43 \pm 0.05$ & $3.42 \pm 0.05$ & $4.53 \pm 0.05$ & $3.20 \pm 0.10$ & $3.18 \pm 0.16$ & 4.63 \\
GJ215 & $3.77 \pm 0.03$ & $3.68 \pm 0.04$ & $4.65 \pm 0.03$ & $3.44 \pm 0.08$ & $3.50 \pm 0.05$ & 4.79 \\
GJ378 & $3.63 \pm 0.04$ & $3.65 \pm 0.04$ & $4.53 \pm 0.06$ & $3.41 \pm 0.05$ & $3.44 \pm 0.04$ & 4.68 \\
GJ699 & $3.20 \pm 0.10$ & $3.25 \pm 0.10$ & $4.17 \pm 0.16$ & $3.01 \pm 0.10$ & $2.98 \pm 0.10$ & 4.30 \\
GJ701 & $3.62 \pm 0.06$ & $3.61 \pm 0.07$ & $4.74 \pm 0.03$ & $3.44 \pm 0.11$ & $3.42 \pm 0.11$ & 4.84 \\
GJ725A & $3.59 \pm 0.07$ & $3.53 \pm 0.07$ & $4.53 \pm 0.12$ & $3.37 \pm 0.03$ & $3.35 \pm 0.03$ & 4.66 \\
GJ880 & $3.53 \pm 0.02$ & $3.48 \pm 0.03$ & $4.40 \pm 0.03$ & $3.30 \pm 0.04$ & $3.43 \pm 0.03$ & 4.56 \\
GJ908 & $3.38 \pm 0.06$ & $3.37 \pm 0.07$ & $4.42 \pm 0.10$ & $3.14 \pm 0.09$ & $3.35 \pm 0.07$ & 4.54 \\
LHS178 & $3.35 \pm 0.09$ & $3.43 \pm 0.07$ & $4.35 \pm 0.06$ & $3.34 \pm 0.10$ & $3.19 \pm 0.15$ & 4.49 \\
LHS1226 & $3.75 \pm 0.05$ & $3.71 \pm 0.05$ & $4.69 \pm 0.08$ & $3.52 \pm 0.06$ & $3.62 \pm 0.06$ & 4.83 \\
LHS2018 & $3.40 \pm 0.04$ & $3.62 \pm 0.03$ & $4.51 \pm 0.03$ & $3.32 \pm 0.04$ & $3.50 \pm 0.04$ & 4.65 \\
\hline
\end{tabular}

Table 8

Isotopic Fractions $\mathrm{f}(i)={ }^{i} \mathrm{Ti} / \Sigma^{i} \mathrm{Ti}$

\begin{tabular}{|c|c|c|c|c|c|c|}
\hline Star & {$[\mathrm{Fe} / \mathrm{H}]$} & $f(46)$ & $\mathrm{f}(47)$ & $f(48)$ & $f(49)$ & $\mathrm{f}(50)$ \\
\hline GJ184 & -0.5 & $0.064 \pm 0.009$ & $0.062 \pm 0.010$ & $0.801 \pm 0.098$ & $0.037 \pm 0.016$ & $0.036 \pm 0.017$ \\
\hline GJ215 & -0.1 & $0.096 \pm 0.008$ & $0.078 \pm 0.011$ & $0.729 \pm 0.060$ & $0.045 \pm 0.013$ & $0.052 \pm 0.015$ \\
\hline GJ378 & -0.4 & $0.089 \pm 0.009$ & $0.093 \pm 0.011$ & $0.707 \pm 0.101$ & $0.054 \pm 0.011$ & $0.057 \pm 0.015$ \\
\hline GJ699 & -0.8 & $0.079 \pm 0.018$ & $0.088 \pm 0.020$ & $0.735 \pm 0.275$ & $0.051 \pm 0.015$ & $0.047 \pm 0.018$ \\
\hline GJ701 & -0.2 & $0.061 \pm 0.006$ & $0.059 \pm 0.008$ & $0.801 \pm 0.059$ & $0.040 \pm 0.010$ & $0.038 \pm 0.015$ \\
\hline GJ725A & -0.3 & $0.085 \pm 0.014$ & $0.074 \pm 0.014$ & $0.741 \pm 0.199$ & $0.051 \pm 0.013$ & $0.049 \pm 0.017$ \\
\hline GJ880 & 0.0 & $0.094 \pm 0.007$ & $0.083 \pm 0.011$ & $0.694 \pm 0.054$ & $0.055 \pm 0.010$ & $0.074 \pm 0.015$ \\
\hline GJ908 & -0.5 & $0.069 \pm 0.010$ & $0.068 \pm 0.012$ & $0.759 \pm 0.183$ & $0.040 \pm 0.013$ & $0.065 \pm 0.017$ \\
\hline LHS178 & -1.0 & $0.072 \pm 0.014$ & $0.087 \pm 0.016$ & $0.721 \pm 0.107$ & $0.070 \pm 0.018$ & $0.050 \pm 0.021$ \\
\hline LHS1226 & -0.1 & $0.084 \pm 0.010$ & $0.076 \pm 0.011$ & $0.729 \pm 0.142$ & $0.049 \pm 0.011$ & $0.062 \pm 0.016$ \\
\hline LHS2018 & -0.5 & $0.057 \pm 0.009$ & $0.094 \pm 0.010$ & $0.731 \pm 0.053$ & $0.047 \pm 0.010$ & $0.071 \pm 0.015$ \\
\hline
\end{tabular}

\section{DISCUSSION}

\subsection{Observed Trends}

Our results are summarized in Figures 7 and 8. In Figure 7, we display the isotopic fractions ${ }^{i} \mathrm{Ti} /{ }^{48} \mathrm{Ti}$ as a function of $[\mathrm{Fe} / \mathrm{H}]$ with the different symbols denoting thin (open squares), thick (filled circles) disk stars, and the cross the halo star LHS178. The three stars with kinematics that do not allow a clean attribution to either the thin or thick disk are represented by half-filled circles. In Figure 8, we display ratios among the less abundant isotopes with ${ }^{46} \mathrm{Ti}$ in the denominator, and also the ratio ${ }^{46} \mathrm{Ti} /{ }^{48} \mathrm{Ti}$.

Three statements suffice to summarize the results in Figure 7. First, the stars with $[\mathrm{Fe} / \mathrm{H}] \sim 0$ display the solar system isotopic ratios, as expected by every other abundance measure for the 


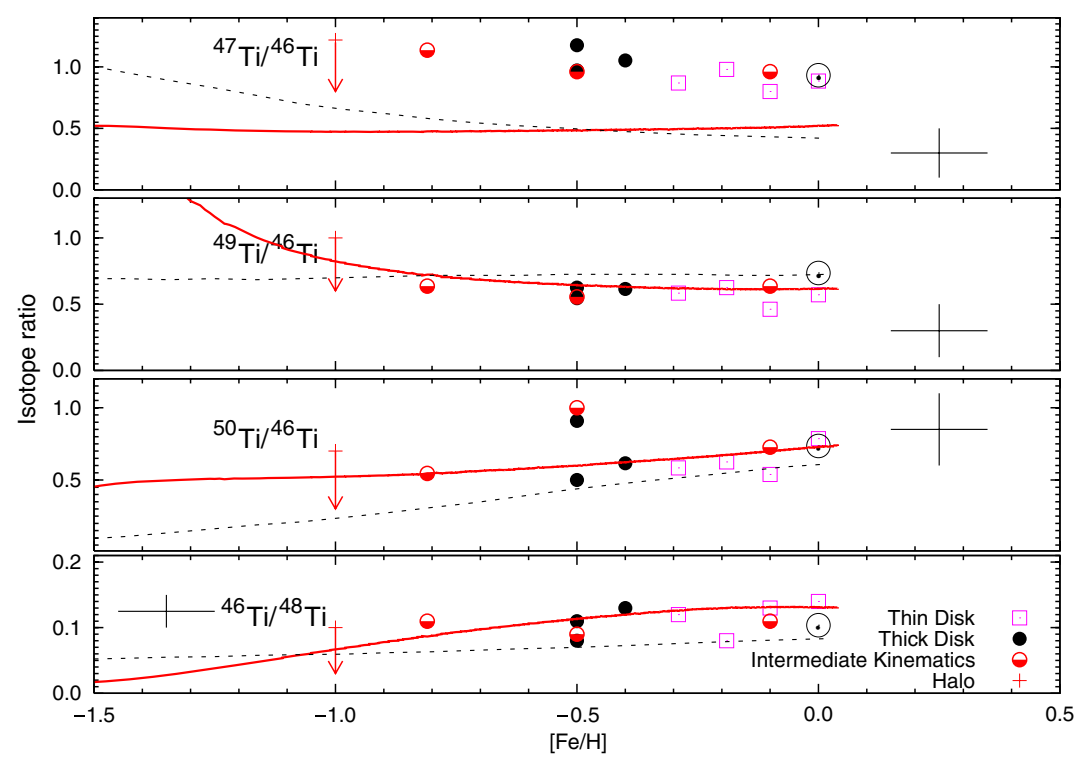

Figure 8. Observed and predicted isotopic ratios ${ }^{47} \mathrm{Ti} /{ }^{46} \mathrm{Ti},{ }^{49} \mathrm{Ti} /{ }^{46} \mathrm{Ti},{ }^{50} \mathrm{Ti} /{ }^{46} \mathrm{Ti}$, and ${ }^{46} \mathrm{Ti} /{ }^{48} \mathrm{Ti}$. Stellar populations of our stars are represented as follows: thick disk $=$ filled circles, thin disk $=$ unfilled squares, halo $=$ cross (arrow indicates an upper limit), and thick-thin disk $=$ intermediate kinematics $=$ half-filled circle. Predictions are from N. Prantzos (2008, private communication; red line) and C. Kobayashi (2008, private communication; black dashed line). An indication of representative uncertainty is given by the cross in each panel. Solar system ratios are denoted at $[\mathrm{Fe} / \mathrm{H}]=0$ by the standard symbol for the Sun.

(A color version of this figure is available in the online journal.)

Sun and stars of solar metallicity; mismatches between the GCE predictions and the solar system abundances are not attributable to the latter being anomalous. Second, there is no clear difference between isotopic abundances for thin and thick disk stars across this small sample. Third, the isotopic ratios are sensibly constant over the observed metallicity range from $[\mathrm{Fe} / \mathrm{H}]$ of zero to about -0.8 . A similar set of conclusions applies to Figure 8 where ratios with respect to ${ }^{46} \mathrm{Ti}$ are given.

\subsection{Nucleosynthesis Predictions}

Pioneering predictions of the variation of the Ti isotopic ratios with $[\mathrm{Fe} / \mathrm{H}]$ were provided by TWW95: relative to ${ }^{48} \mathrm{Ti}$ and the solar system isotopic ratios, the predictions for ${ }^{46} \mathrm{Ti},{ }^{47} \mathrm{Ti},{ }^{49} \mathrm{Ti}$, and ${ }^{50} \mathrm{Ti}$ were factors of 2 too large, 3 too small, spot on, and 2 too small, respectively. Isotopic ratios were predicted to decline steeply with decreasing $[\mathrm{Fe} / \mathrm{H}]$ : declines by factors of 8 for ${ }^{46} \mathrm{Ti}$, 6 for ${ }^{47} \mathrm{Ti}, 2$ for ${ }^{49} \mathrm{Ti}$, and 30 for ${ }^{50} \mathrm{Ti}$ between $[\mathrm{Fe} / \mathrm{H}]=0$ and -1 .

Such predictions do not match our results terribly well and, in particular, the $[\mathrm{Fe} / \mathrm{H}]$ dependences appear at odds with the observations. More recent predictions provide a closer fit to the observations and given the complexity of calculations of nucleosynthetic yields by Type II and Ia supernovae and certain ingredients in a GCE recipe (i.e., the initial mass function or IMF), factors of 2 agreement between prediction and observation should be considered a success. Two recent predictions are shown in Figure 7: (1) GCE as represented by K06 with data supplied by C. Kobayashi (2008, private communication), and (2) GCE as modeled by GP00 with updates contributed by N. Prantzos (2008, private communication). Before remarking on the comparison between these two predictions and our observations, we discuss the yields of the five $\mathrm{Ti}$ isotopes from both types of supernovae.

Isotopes of titanium are synthesized in both Type II and Type Ia supernovae. Predictions of the evolution of the isotopic abundances with metallicity involve calculations of the relative frequencies of Type II and Ia supernovae and their respective yields plus a gaggle of additional assumptions about stars (e.g., the IMF) and the Galaxy (e.g., infall rates). Our intent here is not to diagnose critically the published predictions for the relative isotopic abundances but rather to extract from published predictions aspects of the nucleosynthesis of the Ti isotopes.

K06 (their Table 3) present yields (in solar masses) from stellar generations of Type II supernovae and hypernovae with initial metal mass fractions for $Z=0.0,0.001,0.004$, and 0.02 . The predicted yields expressed as number density ratios are ${ }^{46} \mathrm{Ti} /{ }^{48} \mathrm{Ti}=0.055(0.093),{ }^{47} \mathrm{Ti} /{ }^{48} \mathrm{Ti}=0.055(0.050),{ }^{49} \mathrm{Ti} /$ ${ }^{48} \mathrm{Ti}=0.035(0.052)$, and ${ }^{50} \mathrm{Ti} /{ }^{48} \mathrm{Ti}=0.004(0.053)$ for initial compositions $Z=0.001$ and in parentheses $Z=0.02$. (The value $Z=0.02$ is now a suprasolar value: the solar value is $Z=0.012$ according to Asplund et al. 2005.) Apart from the case of ${ }^{50} \mathrm{Ti}$, the yields relative to ${ }^{48} \mathrm{Ti}$ are weakly dependent on the initial $Z$ but ${ }^{50} \mathrm{Ti}$ relative to ${ }^{48} \mathrm{Ti}$ is produced below the $1 \%$ level for $Z$ less than about 0.003 .

Other calculations of Type II supernovae yields predict a steeper rise in the isotopic ratios with increasing $Z$. Woosley \& Weaver (1995) and Chieffi \& Limongi (2004) presented yields in ejecta as a function of $Z$ after integration over an IMF (with other assumptions) showing a factor of about 10 increase for ${ }^{46} \mathrm{Ti}$ and ${ }^{47} \mathrm{Ti}$, a factor of 2 increase for ${ }^{49} \mathrm{Ti}$, and a factor of 30 increase for ${ }^{50} \mathrm{Ti}$ (all relative to ${ }^{48} \mathrm{Ti}$ ) from $Z=0.1 Z_{\odot}$ to $Z=Z_{\odot}$ (N. Prantzos 2008, private communication). For ${ }^{46} \mathrm{Ti}$ and ${ }^{47} \mathrm{Ti}$, these increases are much larger than those given by K06.

Type Ia supernovae also contribute to the Ti abundances. For the yields from Type Ia supernovae, TWW95 took the model W7 for all $Z$ with the nucleosynthesis as calculated by Thielemann et al. (1986): W7 is a popular model (Nomoto et al. 1984). This model gives ratios with respect to ${ }^{48} \mathrm{Ti}$ of $0.099\left({ }^{46} \mathrm{Ti}\right), 0.0016$ $\left({ }^{47} \mathrm{Ti}\right), 0.046\left({ }^{49} \mathrm{Ti}\right)$, and $0.0077\left({ }^{50} \mathrm{Ti}\right) . \mathrm{K} 06$ 's calculations also adopt the model W7 for all $Z$ but with the ejecta's composition as calculated by Nomoto et al. $(1984,1997)$ : the ejecta has ratios with respect to ${ }^{48} \mathrm{Ti}$ of $0.088\left({ }^{46} \mathrm{Ti}\right), 0.0030\left({ }^{47} \mathrm{Ti}\right), 0.082\left({ }^{49} \mathrm{Ti}\right)$, and $0.060\left({ }^{50} \mathrm{Ti}\right)$. Note the nearly tenfold increase in the ${ }^{50} \mathrm{Ti}$ relative abundance over TWW95's adopted value. 
The predictions by Prantzos in Figure 7 are based on the prescription for GCE described by GP00 who took yields for Type II supernovae from TWW95. For the predictions in Figures 7 and 8, Prantzos took yields from Chieffi \& Limongi (2004) but the changes in the isotopic ratios attributable to the switch in Type II supernovae yields are slight. GP00 adopted yields for Type Ia supernovae from Iwamoto et al. (1999) for two models: W7 and W70. The former assumes the exploding white dwarf evolved from a star of solar $Z$ and the latter that the white dwarf's progenitor had $Z=0$. For their GCE calculations, GP00 interpolate linearly in $Z$ to obtain yields as a function of $Z$. The W70 (W7) yields provide distinctly non-solar isotopic fractions: ${ }^{46} \mathrm{Ti} /{ }^{48} \mathrm{Ti}=0.0011(0.068),{ }^{47} \mathrm{Ti} /{ }^{48} \mathrm{Ti}=0.0013(0.0025),{ }^{49} \mathrm{Ti} /$ ${ }^{48} \mathrm{Ti}=0.0092(0.082)$, and ${ }^{50} \mathrm{Ti} /{ }^{48} \mathrm{Ti}=0.31(0.52)$. Note the high relative abundance of ${ }^{50} \mathrm{Ti}$ for $\mathrm{W} 7$ from Nomoto et al. in contrast to the lower abundances for the same model adopted by TWW95 and K06. One supposes that the ${ }^{50} \mathrm{Ti}$ yield is critically dependent on some adopted (and changing) nuclear reaction rates in the reaction network.

Hughes et al. (2008) predicted the evolution of the titanium isotopic ratios for a dual-infall model. In this model, the halo forms by infall of primordial gas with the disk formation and evolution accompanied by a second infall episode with gas enriched by ejecta from halo stars. The principal effect of this second episode on the predicted evolution of the titanium isotopes is to increase the ${ }^{50} \mathrm{Ti} /{ }^{48} \mathrm{Ti}$ ratio in the disk. This increase is presumably due to ${ }^{50} \mathrm{Ti}$ production from Type Ia supernovae because their model assumes a delay in the onset of the infall. The resultant predicted isotopic ratios are quite similar to those by Prantzos shown in Figures 7 and 8. (Hughes et al. adopt the Nomoto et al. (1997) yields for Type Ia supernovae.)

These GCE models neglect contributions from AGB stars, low-mass stars that do not die as supernovae but provide $s$ process and other products. Operation of the neutron-capture $s$-process provides not only "heavy" nuclides (e.g., $\mathrm{Sr}$ and Ba) but also redistributes the nuclides such as the Ti isotopes lighter than the Fe-peak. The Ti isotopes most affected by the $s$-process in AGB stars appear to be ${ }^{49} \mathrm{Ti}$ and ${ }^{50} \mathrm{Ti} ;{ }^{50} \mathrm{Ti}$ is neutron magic $(N=28)$ with a small neutron-capture cross section and, thus, is raised in abundance by the $s$-process. Calculations reported by Lugaro et al. (1999) for a solar metallicity AGB star show that the production factor of ${ }^{50} \mathrm{Ti}$ is less than 10 for a production factor of $s$-process "heavy" nuclides of 200-300. Thus, addition of AGB stars to a GCE prescription to account for the abundances of $s$-process products such as $\mathrm{Sr}$ and $\mathrm{Ba}$ is not expected to result in major alterations to the $\mathrm{Ti}$ isotopic ratios predicted by TWW95, GP00, K06, and Hughes et al. (2008). Modifications of Ti isotopic ratios attributed to $s$-process operation in AGB stars are seen in presolar $\mathrm{SiC}$ grains (Lugaro et al. 1999; Huss $\&$ Smith 2007).

\subsection{Observed Trends and Predictions}

Observed ratios ${ }^{i} \mathrm{Ti} /{ }^{48} \mathrm{Ti}$ and ${ }^{i} \mathrm{Ti} /{ }^{46} \mathrm{Ti}$ are compared in Figures 7 and 8, respectively, with the recent predictions from Kobayashi and Prantzos. The predictions account rather well for the observations. Indeed, the latter may be said to fit three of the four ratios in Figure 7 well with the exception being the ${ }^{47} \mathrm{Ti} /{ }^{48} \mathrm{Ti}$ ratio that is underpredicted by a factor of 2 or less. The former consistently underpredict the abundances of the lesser abundant isotopes relative to ${ }^{48} \mathrm{Ti}$ but, except again for ${ }^{47} \mathrm{Ti} /{ }^{48} \mathrm{Ti}$, are within a factor of 2 of the measured ratios. In summary, the recent predictions are an improvement on those by TWW95 mentioned in introductions to the paper and the discussion. These improvements are largely a reflection of changes to the adopted yields. The relatively poor fit in Figure 7 to the observed ${ }^{47} \mathrm{Ti} /{ }^{48} \mathrm{Ti}$ ratios presumably arises from a too low prediction for the yield of ${ }^{47} \mathrm{Ti}$ from Type II supernovae, primarily. All in all, the degree of concordance in Figures 7 and 8 between prediction and observation is a pleasing achievement.

Dissection of the reasons for the now (slight) disagreements between observed and predicted isotopic ratios must ultimately account for the larger failure of the GCE models to predict the observed run of $[\mathrm{Ti} / \mathrm{Fe}]$ with $[\mathrm{Fe} / \mathrm{H}]$. The predictions, as noted in the Introduction, match quite well the shape of the observed run but predict values of $[\mathrm{Ti} / \mathrm{Fe}]$ that are about 0.4 dex less than observed at all $[\mathrm{Fe} / \mathrm{H}]$. A straight increase in yields of ${ }^{48} \mathrm{Ti}$ but not other isotopes quite obviously will completely destroy the agreement between the predicted and observed isotopic ratios.

The simplest empirical solution to this discrepancy for $[\mathrm{Fe} / \mathrm{H}]$ $\leqslant-1$ is to suppose that production of Ti by Type II supernovae has been underestimated by about 0.4 dex. Constraints on this suggestion could be provided were the isotopic ratios known for these metal-poor stars (see below). For $[\mathrm{Fe} / \mathrm{H}] \geqslant-1$, both Type II and Type Ia supernovae contribute to the $[\mathrm{Ti} / \mathrm{Fe}]$ versus $[\mathrm{Fe} / \mathrm{H}]$ relation. In this regime, enhanced Ti production must be achieved with ${ }^{48} \mathrm{Ti}$, the dominant isotope, but no more than modest changes in the yields of the less abundant isotopes can be tolerated if the measured isotope ratios in Figures 7 and 8 are to also fit. Is the present underproduction of ${ }^{47} \mathrm{Ti}$ a clue to how to make up the 0.4 dex discrepancy? In addition, theoretical proposals for achieving enhanced yields of Ti cannot seriously affect presently predicted yields of $\mathrm{Mg}, \mathrm{Si}$, and $\mathrm{Ca}$ for which GCE models (e.g., GP00 and K06) reproduce well their variation of $[$ Element $/ \mathrm{Fe}]$ versus $[\mathrm{Fe} / \mathrm{H}]$. The examples of $\mathrm{Mg}, \mathrm{Si}$, and $\mathrm{Ca}$ also preclude the most naive way to reconcile the observed and predicted runs of $[\mathrm{Ti} / \mathrm{Fe}]$ with $[\mathrm{Fe} / \mathrm{H}]$, i.e., invocation of a 0.4 dex reduction in the yield of Fe from Type II supernovae. Theoreticians may ask-Is it at all possible that there is a systematic error in the $[\mathrm{Ti} / \mathrm{Fe}]$ estimates yet to be uncovered? This seems unlikely given that very similar results are obtained from samples of dwarfs and giants.

\section{CONCLUDING REMARKS}

Several potential observational tests of GCE and stellar nucleosynthesis afforded by measurements of titanium isotopic ratios are left unexplored by our initial foray. Exploration calls for accurate measurement not only of the titanium isotopic ratios but also of the metallicity. The key to accurate metallicities is surely through observation and analysis of infrared $(\mathrm{J}, \mathrm{H}$, and K band) spectra, as Martinache et al. (2009) and Rojas-Ayala \& Lloyd (2009) are about to demonstrate.

Most notably, our observations do not extend to the lower metallicities needed to isolate the contribution from Type II supernovae alone, say $[\mathrm{Fe} / \mathrm{H}]<-1$. In particular, the prediction that the low metallicity Type II supernovae are inefficient producers of ${ }^{50} \mathrm{Ti}$ has not been subjected to a clear test. The isotope ${ }^{50} \mathrm{Ti}$ is present in GJ699 (Barnard's star) to which we assign $[\mathrm{Fe} / \mathrm{H}] \simeq-0.8$ and seemingly too in LHS178 with $[\mathrm{Fe} / \mathrm{H}] \simeq-1$ for which predictions imply a fractional abundance ${ }^{50} \mathrm{Ti} /{ }^{48} \mathrm{Ti}$ of less than about $1 \%$ (Kobayashi) to $3 \%$ (Prantzos) is expected but ${ }^{50} \mathrm{Ti} /{ }^{48} \mathrm{Ti}$ of $6 \%$ is measured for GJ699 and possibly a similar fraction for LHS178.

Exploration of the domain $[\mathrm{Fe} / \mathrm{H}] \leqslant-1$ is highly desirable. The predicted absence of ${ }^{50} \mathrm{Ti}$ needs to be confirmed. The other three less abundant isotopes have predicted abundances of a few percent at $[\mathrm{Fe} / \mathrm{H}]=-2$, and ${ }^{47} \mathrm{Ti}$ has even a $60 \%$ increase 
(relative to ${ }^{48} \mathrm{Ti}$ ) as $[\mathrm{Fe} / \mathrm{H}]$ declines from 0 to -2 in Kobayashi's calculations. At $[\mathrm{Fe} / \mathrm{H}]=-2, \mathrm{~K} 06$ predict ${ }^{46} \mathrm{Ti} /{ }^{48} \mathrm{Ti} \simeq 0.046$, ${ }^{47} \mathrm{Ti} /{ }^{48} \mathrm{Ti} \simeq 0.053$, and ${ }^{49} \mathrm{Ti} /{ }^{48} \mathrm{Ti} \simeq 0.034$ with a negligible amount of ${ }^{50} \mathrm{Ti}\left({ }^{50} \mathrm{Ti} /{ }^{48} \mathrm{Ti}<0.003\right)$. Even at $[\mathrm{Fe} / \mathrm{H}]=-3$, these ratios, ${ }^{50} \mathrm{Ti}$ excepted, are little changed from the values at $[\mathrm{Fe} / \mathrm{H}]=-2$. Prantzos predicts lower relative abundances but also a negligible amount of ${ }^{50} \mathrm{Ti}:{ }^{46} \mathrm{Ti} /{ }^{48} \mathrm{Ti} \simeq 0.013,{ }^{47} \mathrm{Ti} /{ }^{48} \mathrm{Ti}$ $\simeq 0.005,{ }^{49} \mathrm{Ti} /{ }^{48} \mathrm{Ti} \simeq 0.035$ for $[\mathrm{Fe} / \mathrm{H}] \leqslant-1.5$.

Exploration of the range $[\mathrm{Fe} / \mathrm{H}]<-1$ will call for cooler stars than LHS178 in order that lower temperature may compensate for the weaker $\mathrm{TiO}$ bands. Few such targets are yet known. Future discoveries of very metal-poor cool dwarfs will be faint and access to high-resolution spectrographs on very large telescopes will be desirable. In fact, the optimum region for detection and measurement of the ${ }^{50} \mathrm{Ti}$ isotope is likely not the $0-0$ band of the $\gamma$ system that demands high spectral resolution but fortunately, as noted by Clegg et al. (1979), the 0-1 band head of the system for which the ${ }^{50} \mathrm{TiO}$ head falls to the blue of the red-degraded $\mathrm{R}_{3}$ band head by about $3 \AA$ with the ${ }^{49} \mathrm{TiO}$ head midway between the ${ }^{50} \mathrm{TiO}$ and the ${ }^{48} \mathrm{TiO}$ band heads. A full exploitation of this (and other potential indicators of the ${ }^{50} \mathrm{Ti}$ isotope) band head will require ${ }^{48} \mathrm{TiO}$ lines of similar strength to the ${ }^{50} \mathrm{TiO}$ band head. Clegg et al. suggested the $0-0 \delta$ system but other possibilities can most likely be found among the rich set of $\mathrm{TiO}$ electronic systems. Exploration of the spectra of cool subdwarfs will certainly be necessary to find $\mathrm{TiO}$ features cleanly identifiable apart from lines of other molecules, particularly those molecules like $\mathrm{CaH}$ that strengthen relative to $\mathrm{TiO}$ as lower and lower metallicities are encountered.

A project not presently lacking for target stars is the search for isotopic abundance differences between thin and thick stars. This calls for large samples of stars from both populations. The difference in $[\mathrm{Ti} / \mathrm{Fe}]$ at a given $[\mathrm{Fe} / \mathrm{H}]$ in the range of $[\mathrm{Fe} / \mathrm{H}]$ overlap for the two populations is about 0.15 dex from analysis of F and G dwarfs (Reddy et al. 2006). Accurate spectroscopy might reveal a difference in the fractional abundances for these populations. To achieve the necessary accuracy, it will be necessary to observe ${ }^{48} \mathrm{TiO}$ features of a comparable strength to the isotopic lines from the $\gamma$-system's $0-0$ band, as suggested above. Reddy et al. suggest that the difference in $[\mathrm{Ti} / \mathrm{Fe}]$ between the two populations may be largely attributable to the formation of the thin but not thick disk stars from gas contaminated with Type Ia supernovae ejecta. If ${ }^{50} \mathrm{Ti}$ is made in copious amounts (relative to ${ }^{48} \mathrm{Ti}$ ) as some predictions of Type Ia supernovae suggest, the proposed contamination seems difficult to reconcile with the observation here that the thick disk stars have as much, if not more ${ }^{50} \mathrm{Ti}$, as the thin disk stars. However, interpretation is complicated by the prediction that the ${ }^{50} \mathrm{Ti}$ yield from Type II supernovae increases steeply as $[\mathrm{Fe} / \mathrm{H}]$ increases from -1 to higher values. This is just the $[\mathrm{Fe} / \mathrm{H}]$ interval over which Type Ia supernovae make an increasing contribution to composition of the interstellar gas.

We thank Chiaki Kobayashi for providing unpublished predictions for the Galactic chemical evolution of Ti isotopes and Luca Casagrande for sending us photometric estimates of the stellar metallicities. We are especially grateful to Nikos Prantzos for a helpful commentary on the GCE of titanium isotopes and for providing unpublished calculations. We thank Carlos Allende Prieto, Thomas G. Barnes, III, Fritz Benedict, and Chris Sneden for helpful discussions. J.M.C. thanks Jacob Bean and Ian Roederer for assistance and encouragement. We thank the referee for a helpful report. This research has been supported in part by a grant to D.L.L. from the Robert A. Welch Foundation of Houston, Texas.

\section{REFERENCES}

Anders, E., \& Grevesse, N. 1989, Geochim. Cosmochim. Acta, 53, 197

Asplund, M., Grevesse, N., \& Sauval, A. J. 2005, in ASP Conf. Ser. 336, Cosmic Abundances as Records of Stellar Evolution and Nucleosynthesis: In honor of David L. Lambert, ed. T. G. Barnes III \& F. N. Bash (San Francisco, CA: ASP), 25

Bean, J. L., Sneden, C., Hauschildt, P. H., Johns-Krull, C. M., \& Benedict, G. F. 2006, ApJ, 652, 1604

Bensby, T., Feltzing, S., \& Lundström, I. 2003, A\&A, 410, 527

Bensby, T., Feltzing, S., Lundström, I., \& Ilyin, I. 2005, A\&A, 410, 527

Bonfils, X., Delfosse, X., Udry, S., Santos, N. C., Forveille, T., \& Ségransen, D 2005, A\&A, 442, 635

Casagrande, L., Flynn, C., \& Bessell, M. 2008, MNRAS, 389, 585

Chieffi, A., \& Limongi, M. 2004, ApJ, 608, 405

Clegg, R. E. S., Lambert, D. L., \& Bell, R. A. 1979, ApJ, 234, 188

Cutri, R. M., et al. 2003, The IRSA 2MASS All-Sky Point Source Catalog, NASA/IPAC

Davis, S. P., Phillips, J. G., \& Littleton, J. E. 1986, ApJ, 309, 449

Dawson, P. C., \& DeRobertis, M. M. 2004, AJ, 127, 2909

Dehnen, W., \& Binney, J. 1998, MNRAS, 294, 429

Delfosse, X., Forveille, T., Ségransen, D., Beuzit, J.-L., Udry, S., Perrier, C., \& Mayor, M. 2000, A\&A, 364, 217

Gizis, J. E. 1997, AJ, 113, 806

Gliese, W., \& Jahreiß, H. 1991, The Astronomical Data Center CD-ROM Selected Catalogs, I

Goswami, A., \& Prantzos, N. 2000, A\&A, 359, 191

Grupp, F. 2004, A\&A, 420, 289

Hauschildt, P. H., Allard, F., Alexander, D. R., \& Baron, E. 1997, ApJ, 488, 428

Hauschildt, P. H., Allard, F., \& Baron, E. 1999, ApJ, 512, 377

Hughes, G. L., Gibson, B. K., Carigi, L., Sánchez-Bläzquez, P., Chavez, J. M., \& Lambert, D. L. 2008, MNRAS, 390, 1710

Huss, G. R., \& Smith, J. B. 2007, Meteoritics \& Planetary Science, 42, 1055

Iwamoto, K., Brachwitz, F., Nomoto, K., Kishimoto, N., Umeda, H., Hix, W. R., \& Thielemann, F.-K. 1999, ApJS, 125, 439

Johnson, J. A., \& Apps, K. 2009, ApJ, in press (arXiv:0904.3092)

Kobayashi, C., Umeda, H., Nomoto, K., Tominaga, N., \& Ohkubo, T. 2006, ApJ, 653,1145

Kurucz, R. L. 1993, ATLAS9 Stellar Atmosphere Programs and $2 \mathrm{~km} \mathrm{~s}^{-1} \mathrm{Grid}$, Kurucz CD-ROM No. 13 (Cambridge, MA: SAO)

Kurucz, R. L., Furenlid, I., Brault, J., \& Testerman, L. 1984, National Solar Observatory Atlas, NSO

Lambert, D. L., \& Luck, R. E. 1977, ApJ, 211, 443

Lambert, D. L., \& Mallia, E. A. 1972, MNRAS, 156, 337

Lodders, K. 2003, ApJ, 591, 1220

Lugaro, M., Zinner, E., Gallino, R., \& Amari, S. 1999, ApJ, 527, 369

Martinache, F., Rojas-Ayala, B., Ireland, M. J., Lloyd, J. P., \& Tuthill, P. G. 2009, ApJ, 695, 1183

Moore, C. E. 1945, A Multiplet Table of Astrophysical Interest, Princeton, The Observatory

Naulin, C., Hedgecock, I. M., \& Costes, M. 1997, Chem. Phys. Lett., 266, 335

Nomoto, K., Iwamoto, K., Nakasato, N., Thielemann, F.-K., Brachwitz, F., Tsujimoto, T., Kubo, Y., \& Kishimoto, N. 1997, Nucl. Phys. A, 621, 467

Nomoto, K., Thielemann, F.-K., \& Yokoi, K. 1984, ApJ, 286, 644

Perryman, M. A. C., et al. 1997, A\&A, 323, L49

Plez, B. 1998, A\&A, 337, 495

Ramírez, I., Allende Prieto, C., \& Lambert, D. L. 2007, A\&A, 465, 271

Reddy, B. E., Lambert, D. L., \& Allende Prieto, C. 2006, MNRAS, 367, 1329

Reid, I. N., Gizis, J. E., \& Hawley, S. L. 2002, AJ, 124, 2721

Reid, I. N., \& Hawley, S. L. 2005, New Light on Dark Stars: Red Dwarfs, Low-Mass Stars, Brown Dwarfs (Chichester: Praxis Publishing)

Reid, I. N., Hawley, S. L., \& Gizis, J. E. 1995, AJ, 110, 1838

Reiners, A. 2007, A\&A, 467, 259

Rojas-Ayala, B., \& Lloyd, J. P. 2009, in AIP Conf. Proc. 1094, Cool Stars, Stellar Systems and the Sun: 15th Cambridge Workshop, ed. E. Stempels (Melville, NY: AIP), 776

Sneden, C. 1973, PhD thesis, Univ. Texas at Austin

Thielemann, F.-K., Nomoto, K., \& Yokoi, K. 1986, A\&A, 158, 17

Timmes, F. X., Woosley, S. E., \& Weaver, T. A. 1995, ApJS, 98, 617

Tull, R. G., MacQueen, P. J., Sneden, C., \& Lambert, D. L. 1995, PASP, 107, 251

Woolf, V. M., \& Wallerstein, G. 2005, MNRAS, 365, 963

Woosley, S. E., \& Weaver, T. A. 1995, ApJS, 101, 181

Wyckoff, S., \& Wehinger, P. 1972, PASP, 84, 647 